\title{
Direct Fast-Neutron Detection
}

D.C. Stromswold

A.J. Peurrung

R.R. Hansen

P.L. Reeder

Prepared for the U.S. Department of Energy under Contract DE-AC06-76RLO 1830

Pacific Northwest National Laboratory

Richland, Washington 99352 


\section{DISCLAIMER}

This report was prepared as an account of work sponsored by an agency of the United States Government. Neither the United States Government nor any agency thereof, nor any of their employees, make any warranty, express or implied, or assumes any legal liability or responsibility for the accuracy, completeness, or usefulness of any information, apparatus, product, or process disclosed, or represents that its use would not infringe privately owned rights. Reference herein to any specific commercial product, process, or service by trade name, trademark, manufacturer, or otherwise does not necessarily constitute or imply its endorsement, recommendation, or favoring by the United States Government or any agency thereof. The views and opinions of authors expressed herein do not necessarily state or reflect those of the United States Government or any agency thereof. 


\section{DISCLAIMER}

Portions of this document may be illegible in electronic image products. Images are produced from the best available original document. 


\section{Summary}

Direct fast-neutron detection is the detection of fast neutrons before they are moderated to thermal energy. We have investigated two approaches for using proton-recoil in plastic scintillators to detect fast neutrons and distinguish them from gamma-ray interactions. Both approaches use the difference in travel speed between neutrons and gamma rays as the basis for separating the types of events. In the first method, we examined the pulses generated during scattering in a plastic scintillator to see if they provide a means for distinguishing fast-neutron events from gamma-ray events. The slower speed of neutrons compared to gamma rays results in the production of broader pulses when neutrons scatter several times within a plastic scintillator. In contrast, gamma-ray interactions should produce narrow pulses, even if multiple scattering takes place, because the time between successive scatterings is small. Experiments using a fast scintillator confirmed the presence of broader pulses from neutrons than from gamma rays. However, the difference in pulse widths between neutrons and gamma rays using the best commercially available scintillators was not sufficiently large to provide a practical means for distinguishing fast neutrons and gamma rays on a pulse-by-pulse basis. A faster scintillator is needed, and that scintillator might become available in the future. Results of the pulse-width studies were presented in a previous report (Peurrung et al. 1998), and they are only summarized here.

We have also investigated a second method for detecting fast neutrons. This method uses the concept of a "low-density" plastic scintillator to increase the travel distances between scattering events and thus make it easier to distinguish neutrons from gamma rays. In its simplest configuration, this method uses two scintillators separated by an air gap to make a timeof-flight (TOF) measurement. Neutrons (even fast neutrons) will take a significantly longer time to travel across the gap from one scintillator to the other than will gamma rays. In addition, the flight times for neutrons will vary according to the neutron energy. Experimental results show that TOF measurements are capable of distinguishing neutrons sources having sufficiently different energy spectra (in particular, $(\alpha, n)$ reactions on Be with average neutron energy of about $4.5 \mathrm{MeV}$, fission sources with energy about $2 \mathrm{MeV}$, and $(\alpha, n)$ reactions on Li with energy less than $1 \mathrm{MeV}$ ). A possible application for the TOF measurement is distinguishing between $\mathrm{Pu}$ metal (spontaneous fission neutrons) and $\mathrm{PuO}_{2}$ (spontaneuous fission and $(\alpha, n)$ ) in weapons component inspection. TOF measurements on a small weapons-grade $\mathrm{PuO}_{2}$ source showed a clear difference from a ${ }^{252} \mathrm{Cf}$ source (spontaneous fission) spectrum. The detection efficiency for neutrons from a $\mathrm{PuBe}$ or ${ }^{252} \mathrm{Cf}$ source was about 1 percent for those neutrons that were incident on the first of two plastic scintillators $(30 \times 30 \mathrm{~cm})$ separated by a $20-\mathrm{cm}$ air gap. The detection efficiency for fast neutrons from $\mathrm{PuBe}$ or ${ }^{252} \mathrm{Cf}$ was about 100 times more than from low-energyneutron sources such as PuLi. This suggests that TOF could be useful in counting inducedfission neutrons from uranium in the presence of an active source of low-energy neutrons.

\section{Reference}

AJ Peurrung, RR Hansen, PL Reeder, and DC Stromswold. 1998. Direct Fast-Neutron Detection: a Progress Report, PNNL-11994, Pacific Northwest National Laboratory, Richland, Washington. 



\section{Contents}

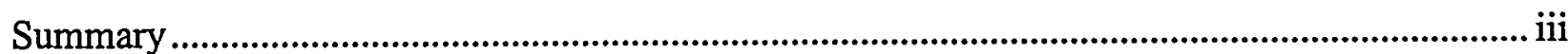

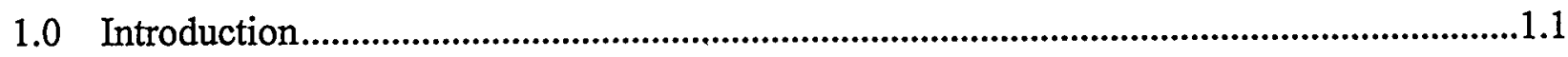

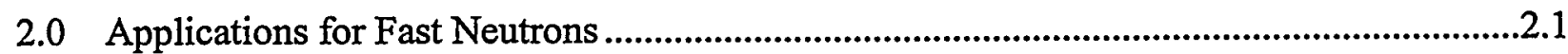

3.0 Pulse Shape Analysis ..........................................................................................................

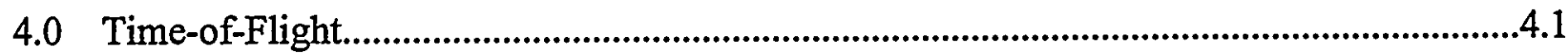

4.1 Detailed Analysis of TOF Spectra .............................................................................. 4.8

4.2 TOF and Energy Spectra............................................................................................ 4.10

4.3 Fast-Neutron Detection Efficiency ................................................................................ 4.14

4.4 Analysis of TOF versus PMT Multiplicity .................................................................. 4.17

5.0 Possible Future Work .........................................................................................................

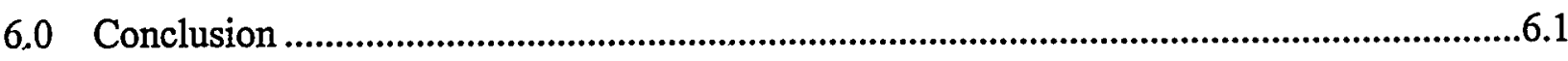

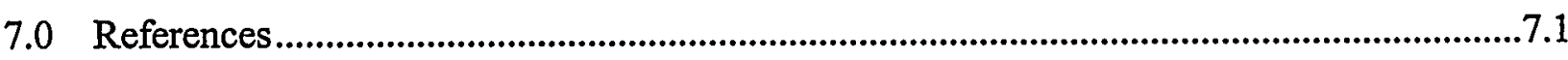

\section{Figures}

Figure 3.1. Electronic Setup for Measuring Pulse Shapes for Gamma-Ray and Neutron

Interactions in Plastic Scintillator ............................................................................................ 3.1

Figure 3.2. Gamma-Ray Pulses (sorted by increasing pulse width) ............................................ 3.2

Figure 3.3. Neutron Pulses (sorted by increasing pulse width)...................................................... 3.2

Figure 3.4. Cerenkov Pulses Illustrating Narrow Pulse Widths.................................................... 3.3

Figure 4.1. Two Plastic Scintillator Blocks Separated by an Air Gap ......................................... 4.1

Figure 4.2. Time-of-Flight Spectrum for Gamma Rays from ${ }^{137} \mathrm{Cs}$........................................... 4.3

Figure 4.3. Time-of-Flight Spectrum for Neutrons and Gamma Rays from ${ }^{239} \mathrm{PuBe}$................ 4.4

Figure 4.4. Gamma-Rays Appearing as "False Neutron" Events ................................................ 4.4

Figure 4.5. Neutron Detection Efficiency Dependence on Time Threshold and Number of

Tubes Detecting Events ............................................................................................................ 4.5

Figure 4.6. Time-of-Flight Spectrum Obtained Using PuLi Neutron Source, which Emits

Neutrons with an Average Energy of $0.7 \mathrm{MeV}$...................................................................... 4.6

Figure 4.7. Time-of-Flight Spectrum for PuBe Source, which Emits Neutrons of Average

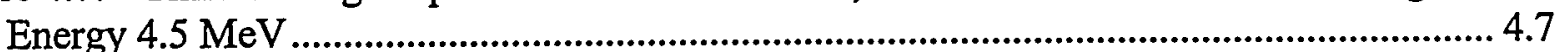

Figure 4.8. TOF Spectra for PuLi.............................................................................................. 4.9

Figure 4.9. TOF Spectra (Normalized) for the ${ }^{252} \mathrm{Cf}$ Source at Three Different

Discriminator Settings .......................................................................................................... 4.10

Figure 4.10. Energy Spectra for Scattered Neutrons from ${ }^{252} \mathrm{Cf}$ Source at Various

Discriminator Settings

Figure 4.11. Energy Spectra for ${ }^{252} \mathrm{Cf}$.................................................................................... 4.11

Figure 4.12. TOF Spectra for $\mathrm{PuBe},{ }^{252} \mathrm{Cf}$, and ${ }^{239} \mathrm{PuO}_{2}$ Sources with Discriminators

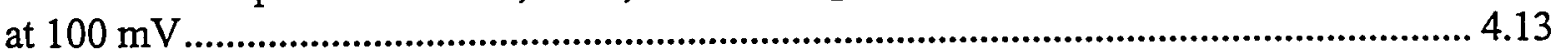

Figure 4.13. Energy Spectra of Scattered Neutrons with Discriminator at $100 \mathrm{mV}$................. 4.14 
Figure 4.14. Energy Spectrum of Scattered Neutrons from AmLi and PuLi Sources with Discriminator at $100 \mathrm{mV}$

Figure 4.15. Fast Neutron Detection Efficiency by Scattering TOF for ${ }^{252} \mathrm{Cf}$ Source with Various

Discriminator Levels.

Figure 4.16. TOF Spectra for $\mathrm{PuBe}$ Source with Discriminator $=100 \mathrm{mV}$

Figure 4.17. TOF Spectra for PuLi Source with Discriminator $=100 \mathrm{mV}$

\section{Tables}

Table 4.1. Neutron-Emitting Sources and Energies.

Table 4.2. Neutron Emission from ${ }^{239} \mathrm{PuO}_{2}$ Source

Table 4.3. Fast Neutron Detection Efficiency by Scattering TOF

Table 4.4. Ratio of 1 PMT Events to 4 PMT Events for Various Sources with Discriminator at $100 \mathrm{mV}$. 


\subsection{Introduction}

Fast neutrons are often detected by first moderating (slowing) them to thermal energies where they can react more readily in certain detectors, such as gas-filled tubes containing ${ }^{3} \mathrm{He}$ or $\mathrm{BF}_{3}$. Fast neutrons are generally slowed by using a hydrogenous moderating material, such as polyethylene, where successive collisions between the neutron and hydrogen atoms efficiently transfer energy away from the neutron. However, during the slowing-down process the information on the original energy of the neutron, its direction of travel, and the time of emission is lost. For many applications, the detection of "fast" neutrons that retain this information is highly desirable.

Plastic scintillators offer one method for detecting fast neutrons. However, gamma rays can also interact in the plastic scintillator, and in mixed neutron and gamma-ray radiation fields, distinguishing neutrons from gamma-rays becomes the pre-eminent task. Our work on fast neutron detection with plastic scintillators uses the difference in speed with which neutrons and gamma rays travel as a means for distinguishing the two types of radiation. The relatively slow speed of even fast neutrons, in comparison with the speed of light at which gamma rays travel, offers a possible mechanism for distinguishing neutrons from gamma rays in plastic scintillators. Neutrons having an energy of $1.0 \mathrm{MeV}$ travel at a speed of $1.4 \mathrm{~cm} / \mathrm{ns}$, whereas gamma rays travel at $30 \mathrm{~cm} / \mathrm{ns}$. The difference in speeds should make it possible to distinguish neutrons that scatter several times within a plastic scintillator from gamma-ray events (whether they scatter once or multiple times). The time interval between successive scatterings of fast neutrons in a single piece of plastic is about $3 \mathrm{~ns}$, whereas for gamma rays successive interactions should occur in less than $1 \mathrm{~ns}$. If the plastic scintillator has a sufficiently short fluorescence lifetime, and the emitted light is detected and converted to an electrical pulse quickly enough, it might be possible to identify neutrons based on multiple scattering. Thus a neutron could be characterized by successive pulses separated in time by about $3 \mathrm{~ns}$. In a real detection system, the successive pulses may appear as a single, broad pulse, which hopefully could be distinguished from a narrow, gamma-ray pulse. This method is a time-of-flight (TOF) measurement, but in an unconventional sense, with very short time intervals being measured in a single scintillator. Our test results using this technique have been presented previously (Peurrung et al. 1997; Peurrung et al. 1998; Reeder et al. 1998; Hansen et al. 1998). These results are summarized in Section 3.0 (Pulse Shape Analysis) of this report. However, no commercial plastic scintillators have been found with a short enough fluorescence lifetime to allow practical separation of fast-neutron and gamma-ray events.

In the absence of a sufficiently fast scintillator to distinguish neutrons and gamma rays using pulse width, we have explored an alternative method that uses a "low-density" scintillator to extend the flight distance and time between successive scatterings. In its simplest configuration, this technique uses two pieces of plastic scintillator separated by an air gap. This method is a traditional TOF measurement, and the scintillators and photomultiplier tubes do not need to have a particularly fast response. With the two separated scintillators, the experimental challenge is to maintain good neutron detection efficiency while achieving sufficient discrimination between neutron and gamma-ray flight times. In Section 4.0 (Time of Flight), we 
describe our experiments to measure the detection efficiency of this detection technique and distinguish between fast-neutron sources having different energy spectra.

Liquid scintillators using pulse-shape analysis are a possible alternative to plastic scintillators for detecting fast neutrons (Knoll 1989). However, the degree of discrimination they offer against gamma rays is inadequate for fission-related events when the gamma-ray flux is large. Also, liquid scintillators produce relatively long pulses, and the performance in high count rates is limited by pulse pileup. 


\subsection{Applications for Fast Neutrons}

The applications of interest for this work involve the detection of fast neutrons emitted from special nuclear material. Some isotopes of plutonium have sufficiently large neutron yields from spontaneous fission that these isotopes can be detected using neutrons. The amount of plutonium can be measured if information on the specific isotopes present is available. Although uranium does not spontaneously fission, it can be induced to fission by the addition of a neutron source. Sources of low-energy neutrons, such as $\mathrm{AmLi}$ with an average energy of about 0.3 $\mathrm{MeV}$, can be used to induce fission in ${ }^{235} \mathrm{U}$. The fast neutrons produced by fission can be distinguished from the source neutrons by their energy if a fast-neutron detector is available and an energy discriminator excludes the low-energy neutrons.

Neutron coincidence counters (Reilly et al. 1991) provide a means for measuring the fission rate from a source. These coincidence counters typically use ${ }^{3} \mathrm{He}$ tubes embedded in polyethylene to count the neutrons after they have been moderated to thermal energy. Although the neutrons are emitted from the source in coincidence, they take about $50 \mu$ s to slow down, and they lose their tight time correlation. As a result, a much wider time window must be used to determine coincidence than would be required if the neutrons were detected directly as fast neutrons (when timing of only a few nanoseconds would be possible). The wide time window necessary when measuring thermal neutrons greatly increases the background from chance coincidences. Segmented fast-neutron detectors can fulfill the functions of the traditional neutron coincidence detectors with greatly reduced accidental coincidence rates.

Pulsed neutron sources, such as accelerators and source shufflers, are also used as interrogating sources to induce fission. The rate of die-away of neutrons after the interrogating source is removed gives a measure of uranium based on the induced fission neutrons. The prompt timing possible through fast-neutron detection should make possible the coincident detection of fission neutrons and allow faster pulse repetition rates. After a few collisions, the interrogating neutrons will have energies less than $0.5 \mathrm{MeV}$ and will not be detected whereas the more energetic fission neutrons will be detected.

The incident direction of fast neutrons can be determined using special configurations of fast-neutron detectors. In contrast, thermal neutrons have lost most of the information about their original direction of travel because of the multiple scattering they undergo in the process of being converted from fast to thermal energy. The directional information inherent in unscattered, fast neutrons can be used to determine the direction to unknown source locations and perhaps also create images of fast-neutron-emitting sources. In general, directional measurements of fast neutrons can be made by recording scattering angles and flight times for the fast neutrons between separated scintillators. Coded-array (Vanier et al. 1995) technology, which uses a mask to create a specific shadow pattern from a point source, may be viable for imaging the distribution of fast-neutron sources. 


\subsection{Pulse Shape Analysis}

This section presents a brief summary of the results of the investigation of individual pulses produced during multiple scattering of neutrons in a single piece of plastic scintillator. A full discussion of the pulse-shape work is contained in a previous report (Peurrung et al. 1998).

Computer simulations with the code MCNP (Briesmeister 1993) indicated that it should be possible to distinguish neutron-related events from gamma events when the neutrons undergo multiple recoils in a piece of plastic scintillator. Neutron events corresponding to multiple scattering within a scintillator should be characterized by broader pulses because they are a convolution of successive, individual pulses separated in time. To determine if these multiple neutron-scattering events could actually be detected based on broadened pulse width, experiments were conducted with fast plastic scintillators. Figure 3.1 shows the experimental arrangement for these tests. A scintillator (BC428, with a decay time constant $\tau=1.4 \mathrm{~ns}$ ) was coupled to a fast photomultiplier tube (PMT; Hamamatsu R2083, $\tau=0.7 \mathrm{~ns}$ ). The scintillator was $5.1 \mathrm{~cm}$ in diameter and $7.6 \mathrm{~cm}$ long. The output pulse from the photomultiplier tube went directly to a digital storage oscilloscope (Tektronix TDS 684B). The individual pulses were captured by the scope and passed to a computer for analysis.

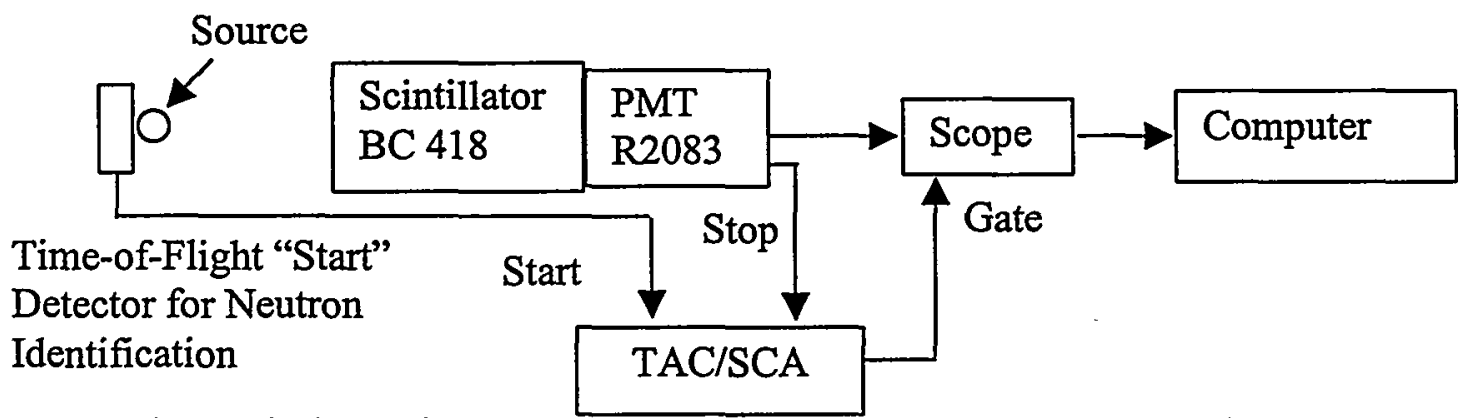

Figure 3.1. Electronic Setup for Measuring Pulse Shapes for Gamma-Ray and Neutron Interactions in Plastic Scintillator. TOF was used to identify neutrons from a ${ }^{252} \mathrm{Cf}$ source.

The principal gamma-ray source used to determine the pulse width was ${ }^{137} \mathrm{Cs}$, and the neutron source was ${ }^{252} \mathrm{Cf}$. To avoid analysis of gamma rays from ${ }^{252} \mathrm{Cf}$, a TOF measurement was performed using an additional scintillator to provide the "start" signal from $\mathrm{a}^{252} \mathrm{Cf}$ fission. Events occurring at a proper time delay (about $10 \mathrm{~ns}$ for a flight distance of $15 \mathrm{~cm}$ ) for neutron travel were analyzed to measure the pulse widths for neutrons. Gamma-ray pulses from the ${ }^{252} \mathrm{Cf}$ source had flight times of only about $0.5 \mathrm{~ns}$.

The computer collected, stored, and calculated the widths of the gamma-ray and neutron pulses. Figure 3.2 shows 100 gamma-ray pulses sorted by increasing pulse width. The pulses in the figure are negative, with a rapid fall and a more gradual rise back to the baseline. Figure 3.3 shows 100 neutron pulses having similar amplitudes to the gamma-ray pulses, again sorted by 
pulse width and plotted on the same time scale as the gamma rays in the previous figure. The gamma-ray and neutron pulses appear quite similar. Because of the relatively small size of the plastic scintillator, only about $10 \%$ of the neutron pulses in Figure 3.3 might be expected to correspond to multiple scattering events of a neutron in the scintillator. Hence, it is reasonable that most of the gamma-ray and neutron pulses have similar widths. In the experimental data, the average width of the widest $10 \%$ of neutron pulses is $5.2 \mathrm{~ns}$. For the gamma-ray pulses, the average width of the widest $10 \%$ pulses was 4.3 ns. This result is consistent with the concept that multiply scattered neutrons should produce wider pulses than the gamma rays. However, the difference in widths does not appear to be sufficient to allow practical identification of neutron events on a pulse-by-pulse basis. When all 100 pulses are averaged, the neutron pulses have a width of $3.5 \mathrm{~ns}$, and the gamma-ray pulses have a width of $3.3 \mathrm{~ns}$.

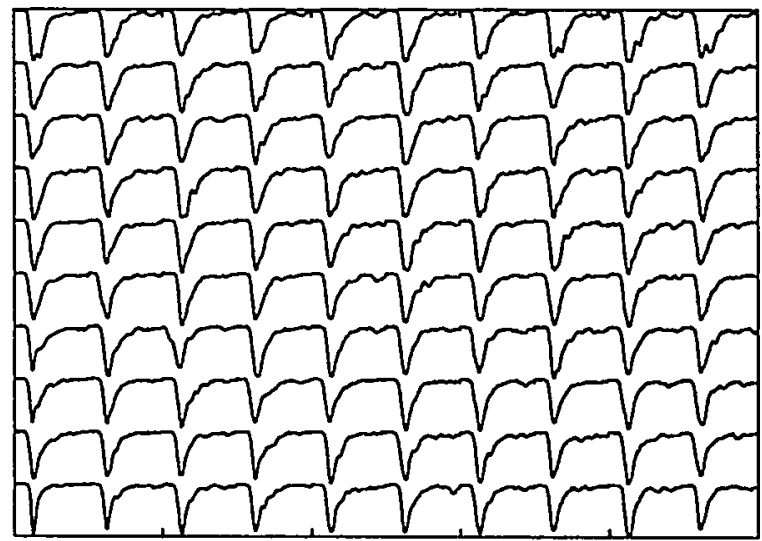

Figure 3.2. Gamma-Ray Pulses (sorted by increasing pulse width)

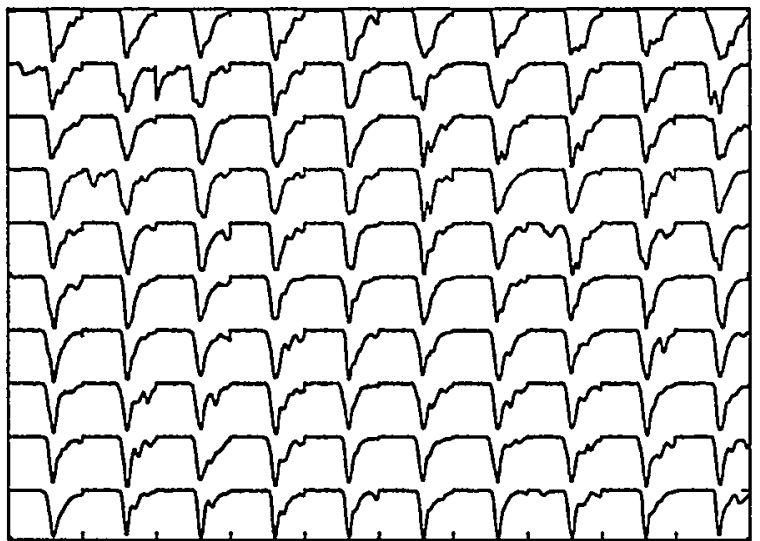

Figure 3.3. Neutron Pulses (sorted by increasing pulse width)

The main difficulty in distinguishing neutron and gamma-ray pulses is that the gammaray pulses are unexpectedly wide. The gamma-ray pulses also show structure (multiple humps). The broadness of the gamma-ray pulses does not appear to be due to the data collection system. This was checked by recording Cerenkov pulses produced by beta particles from ${ }^{90} \mathrm{Sr} /{ }^{90} \mathrm{Y}$ traveling though ordinary (non-scintillator) plastic. Figure 3.4 shows 100 Cerenkov pulses, all of which are narrow, with an average width of $1.4 \mathrm{~ns}$. This width indicates that the signal processing system from the photomultiplier tube on through the remaining electronics is working as expected and not artificially broadening the gamma-ray pulses.

Other scintillators, such as BC422 and quenched types, have been tested, but they also do not provide the necessary performance to distinguish multiply scattered neutrons from gamma rays on a pulse-by-pulse basis. It appears that a faster scintillator than is now commercially available is needed. Such a scintillator would hopefully produce light quickly enough so that gamma-ray pulses will be significantly shorter than those from multiply scattered neutrons. 


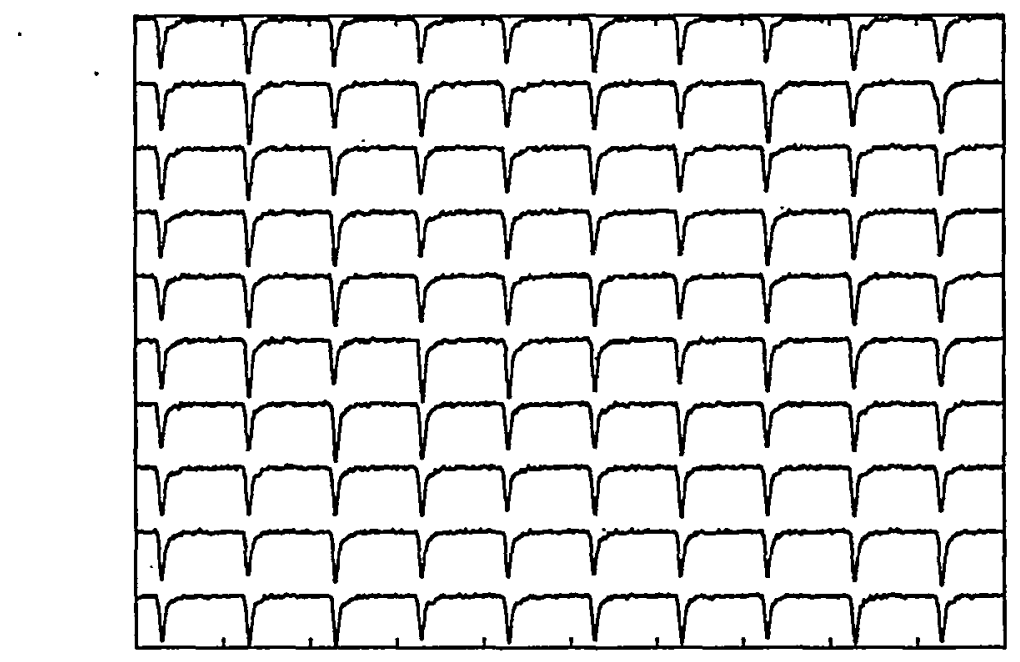

Figure 3.4. Cerenkov Pulses Illustrating Narrow Pulse Widths

3.3 


\subsection{Time-of-Flight}

An alternative approach for distinguishing gamma rays from multiply scattered neutrons is to stretch the neutron interaction time between successive scatterings. One way to do this is to have a "low-density" scintillator array made of two or more plastic scintillator pieces separated by an air gap. Figure 4.1 shows the arrangement of two scintillator blocks separated by a gap. The TOF between the scintillators serves to distinguish neutrons from gamma rays. The scintillators in this arrangement do not need to have a particularly fast response because light is collected separately from each scintillator. Alternative configurations of multiple scintillators are also possible, and they can be optimized for specific applications.

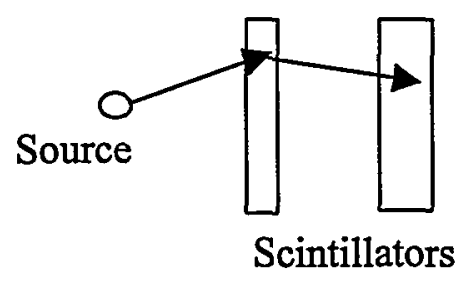

Figure 4.1. Two Plastic Scintillator Blocks Separated by an Air Gap. The TOF between the blocks distinguishes neutrons from gamma rays.

The two-slab scintillator configuration has some inherent directional sensitivity. When neutrons scatter from protons, the permissible scattering angles range from $0^{\circ}$ to $90^{\circ}$ (lab coordinate system). For the configuration shown in Figure 4.1, if the thinner detector is used to start a time-to-amplitude converter (TAC) and the thicker scintillator to stop the TAC, a neutron arriving from the left will give positive time intervals relative to the prompt (gamma-gamma) coincidences. For the same timing conditions, a neutron arriving from the right will give negative time intervals. A neutron arriving from the left can not scatter off a proton in the thicker detector and go back to the thinner detector in a single collision. Likewise a neutron arriving from the right can not scatter from the thinner scintillator back to the thicker one in a single collision. Thus a simple examination of the asymmetry between the number of positive and negative time intervals can indicate from which hemisphere the neutrons arrive. For the TOF measurements, extremely small scattering angles are undesirable because they result in little energy being deposited in the scintillator for detection of the event. Thus the desirable events are those in which the neutron deposits sufficient energy in the first scintillator block for detection, scatters in a direction that allows it to reach the second block, and then scatters in that block, depositing sufficient energy for detection there also.

The neutron's TOF between two plastic scintillators measures the energy of the neutron remaining after the neutron has scattered and deposited part of its energy in the first scintillator. Although the flight time does not directly give the initial energy of the neutron, it can be useful as an indication of the original energy. On average, neutrons with higher initial energy will also have more energy after scattering in the first scintillator than will neutrons with low initial energy. 
Two slabs of BC 408 plastic scintillator $(4 \times 30 \times 30 \mathrm{~cm}$ and $6 \times 30 \times 30 \mathrm{~cm})$ in the arrangement of Figure 4.1 were tested using both neutron and gamma-ray sources. The thicknesses of the two scintillators were chosen based on MCNP modeling to optimize the response to neutrons of energy $2 \mathrm{MeV}$. The greater thickness of the scintillator farthest from the source provides additional stopping power over that of the first scintillator where only scattering was desired. Four photomultiplier tubes (Hamamatsu R329-02) on each scintillator provided the signals for the TOF measurements between the scintillator blocks. In one configuration, the tubes were placed on a flat side $(30 \times 30 \mathrm{~cm})$ of the blocks, and in another configuration, the tubes were on the edges (at the corners that had been cut off to accommodate the tubes). The different positions of the tubes were tested to measure their effects on light-collection efficiency. Data obtained with the photomultiplier tubes on the corners of the blocks showed about $25 \%$ more counts than those with the tubes on the large, flat surfaces.

Signals from each photomultiplier tube were processed through constant fraction discriminators (EG\&G model CF8000, which accepts up to eight inputs). Use of a separate CF8000 for each scintillator block also provided information on the number of tubes detecting an event and the total energy detected in a scintillator. Signals from the constant fraction discriminators were processed by a time-to-amplitude converter that measured the flight time from one scintillator block to the other. For gamma rays, the travel time between two blocks placed $30 \mathrm{~cm}$ apart is about $1 \mathrm{~ns}$, and for neutrons of $1 \mathrm{MeV}$ energy, the travel time is about $22 \mathrm{~ns}$. These times are for the minimum travel path between the blocks, and longer times will occur for travel along a slant path. In addition, neutrons will generally have a range of energies and thus a variety of travel times. From classical mechanics the relationship between neutron energy and velocity is $E_{n}=0.5165 v^{2}$, where $E_{n}$ is neutron energy in $M e V$, and $v$ is the velocity in $\mathrm{cm} / \mathrm{ns}$. For neutron energies of a few $\mathrm{MeV}$, this equation is an acceptable approximation for relating neutron energy and velocity.

Various sources were placed at a distance of $30 \mathrm{~cm}$ from the front face of the thinner scintillator. The distance from the back face of the thinner scintillator to the front face of the thicker scintillator was either $20 \mathrm{~cm}$ or $30 \mathrm{~cm}$. The TAC was started with a pulse from the thicker scintillator (the lower count rate detector) and stopped with a pulse from the thinner scintillator to minimize the rate of start pulses without corresponding stop pulses. This meant that time intervals due to neutrons from the source incident on the thinner detector gave negative time intervals relative to the prompt coincidence peak. The full-scale time range of the TAC was 50 ns. The prompt peak was adjusted using time delays to be centered at Channel 212.5 in a 256-channel pulse height spectrum. The time per channel was measured to be $0.2186 \mathrm{~ns} / \mathrm{ch}$ by use of a time calibrator module (Ortec 462). The minimum energy neutron that could be measured under these conditions was about $0.15 \mathrm{MeV}$, which was below the expected energy required for a neutron to give a pulse in the thicker scintillator.

Figure 4.2 shows a TOF spectrum for gamma rays traveling between two scintillator blocks placed 30-cm apart. (The processed data in this figure shows time increasing from left to right, in contrast with the raw data in which time decreases with increasing channel number because of the reversed start and stop signals.) Note that the gamma-ray peak is well defined and has a full width at half maximum of about 2 ns. Figure 4.3 shows a TOF spectrum for neutrons 
from a ${ }^{239} \mathrm{PuBe}$ source (average energy $4.5 \mathrm{MeV}$ ). This source emits gamma rays as well as neutrons, and hence peaks for both neutrons and gamma rays are present in the TOF spectrum. The neutron events generally occur at the longer flight times, but some gamma-ray events extend into what is predominantly the neutron region of the spectrum. This tail of gamma-ray events creates "false neutron" counts when a source emits both gamma rays and neutrons. The unwanted gamma-ray events can be reduced by raising the time threshold between neutron and gamma-ray events to longer flight times, but doing this also has the effect of reducing the efficiency for counting neutrons.

Figure 4.4 shows how the false neutron counts from a ${ }^{137} \mathrm{Cs}$ source vary with the timethreshold value and with the number of photomultiplier tubes required to detect an event when the scintillator separation distance is $30 \mathrm{~cm}$. Requiring two or more PMTs on a scintillator block to detect an event reduces the.chance for false neutrons. For example, false neutron events are about $10^{-6}$ (i.e., 1 in $10^{6}$ gamma rays incident on the detector is counted as a neutron) for a time threshold of 10 ns (i.e., events occurring with TOF $10 \mathrm{~ns}$ or longer are counted as neutrons) when three or four PMTs detect an event. Requiring multiple PMTs also reduces the detection efficiency for real neutrons as shown in Figure 4.5, which is obtained using data collected for a ${ }^{239} \mathrm{PuBe}$ source. Figure 4.5 shows that neutron counting efficiency (counts from the TOF system per neutron incident on detector) is between 0.004 and 0.011 for a time threshold of $10 \mathrm{~ns}$. For events detected by three or more PMTs, the ratio of neutron detection efficiency to false neutrons is about 7500 (from data in Figures 4.4 and 4.5).

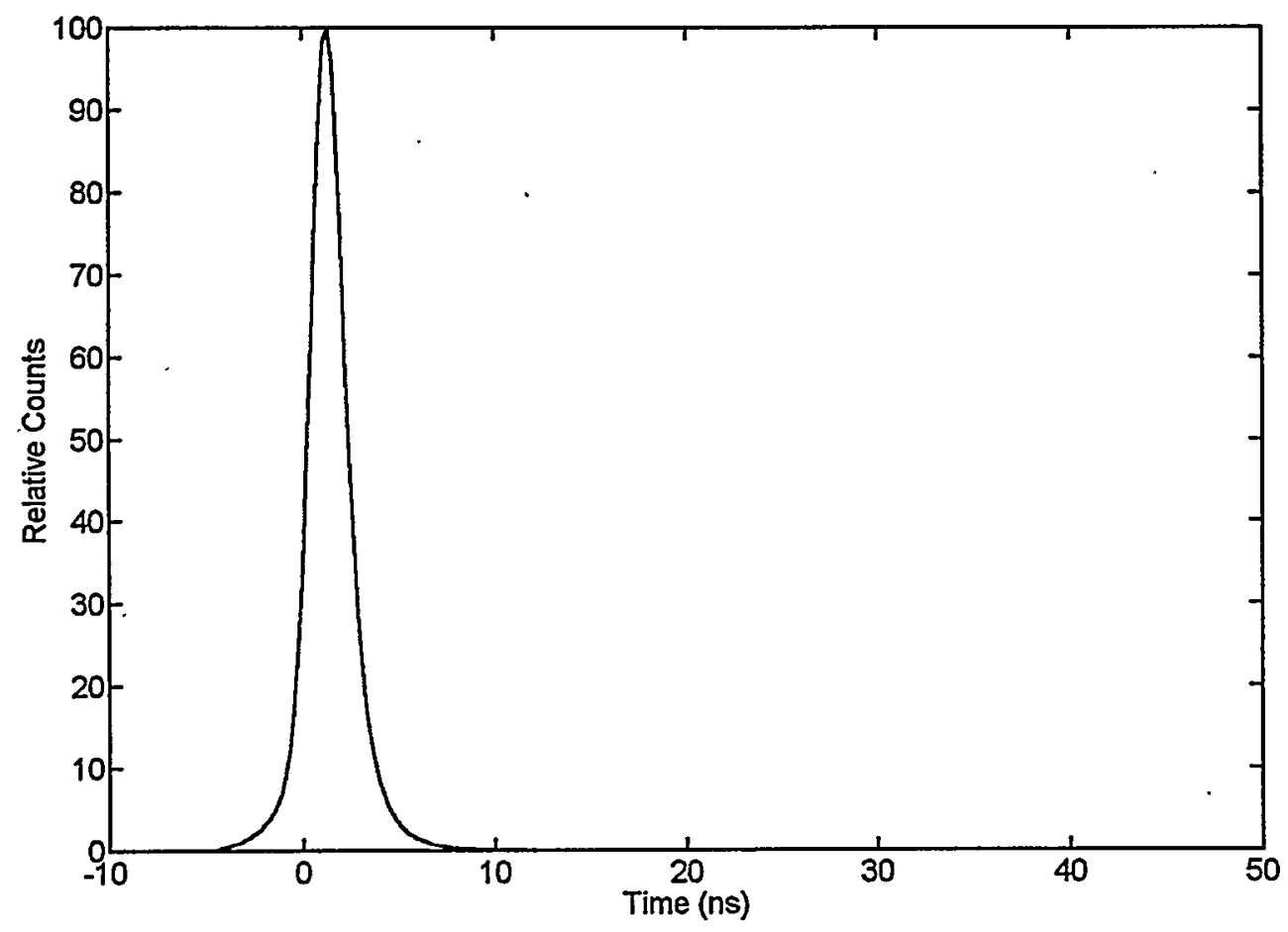

Figure 4.2. Time-of-Flight Spectrum for Gamma Rays from ${ }^{137}$ Cs. Scintillator separation distance $=30 \mathrm{~cm}$. 


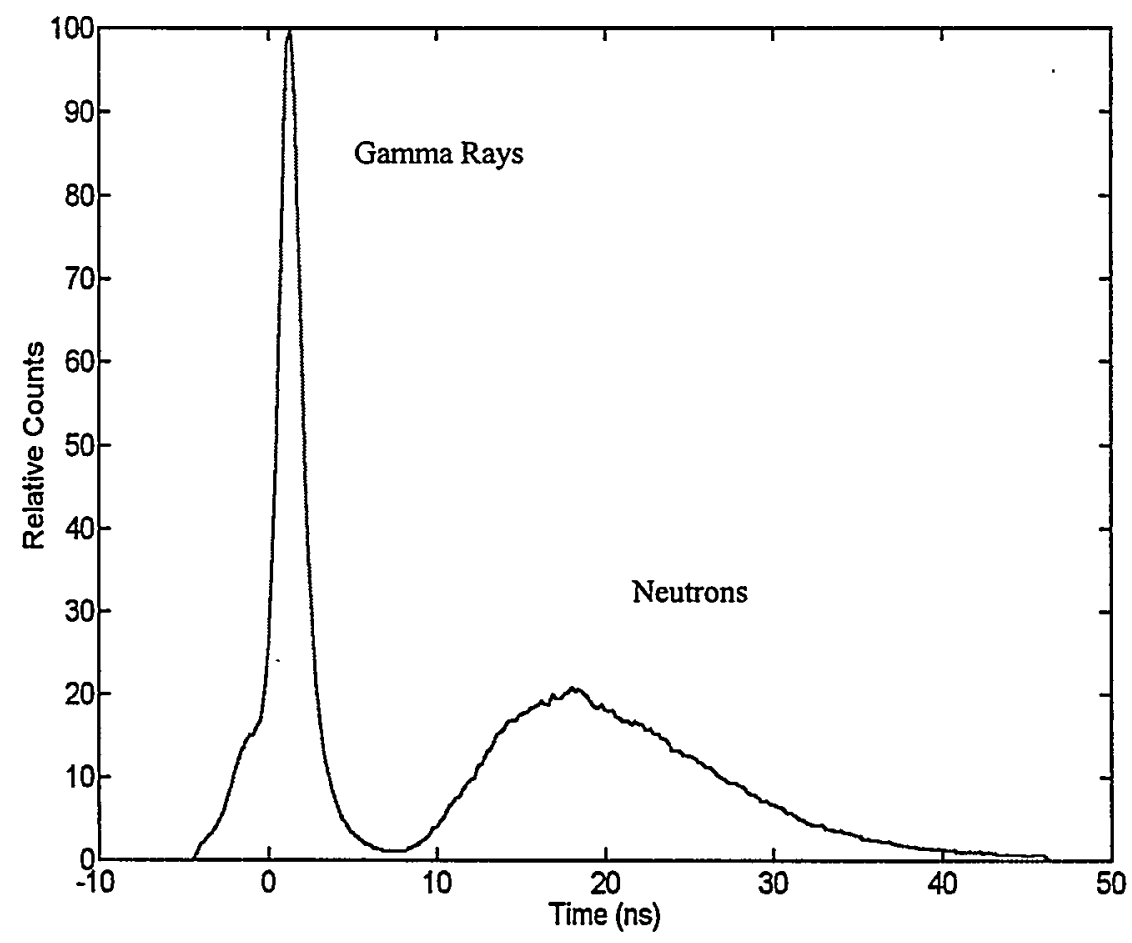

Figure 4.3. Time-of-Flight Spectrum for Neutrons and Gamma Rays from ${ }^{239} \mathrm{PuBe}$. Scintillator separation distance $=30 \mathrm{~cm}$.

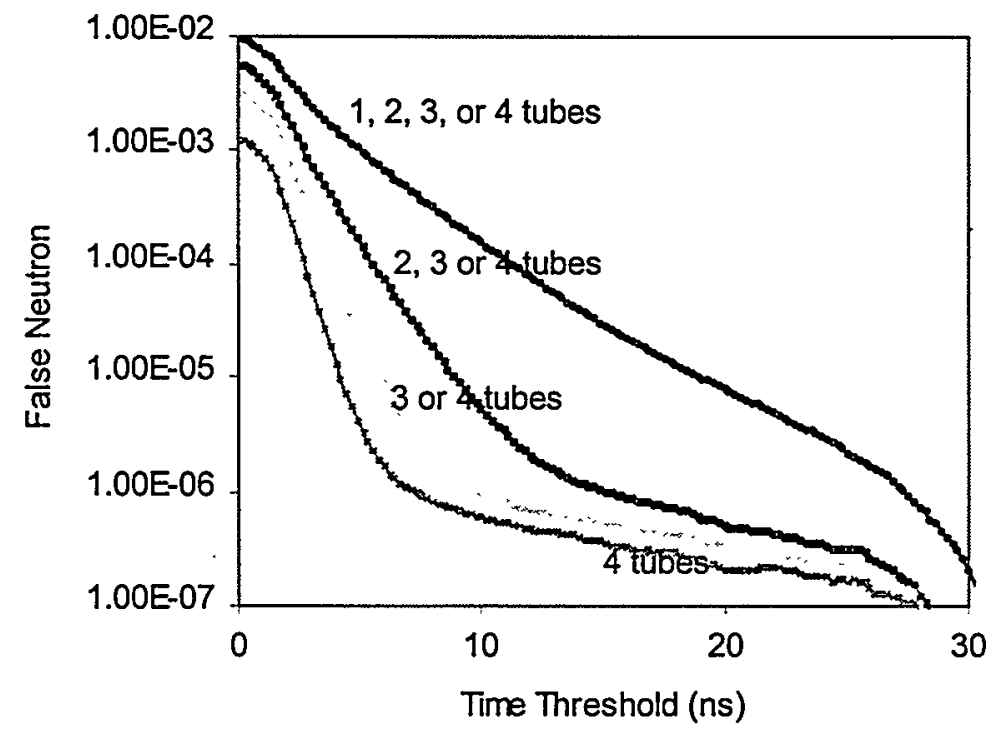

Figure 4.4. Gamma-Rays Appearing as "False Neutron" Events. The time threshold is the time cutoff above which events are considered to be neutron-related because of their long TOF. Fewer false neutrons occur when multiple photomultiplier tubes detect events. 
Moving the scintillator blocks closer together increased the neutron detection efficiency while making it more difficult to discriminate against gamma rays. When the blocks were located only $20 \mathrm{~cm}$ apart and using a time threshold of $10 \mathrm{~ns}$, the neutron detection efficiency obtained using a PuBe source was 0.03 , and the false neutron event rate was about $5 \times 10^{-5}$ (compared to $10^{-6}$ for $30-\mathrm{cm}$ separation).

The performance of the two-slab scintillator system was also tested using a ${ }^{238} \mathrm{PuLi}$ neutron source, which has an average neutron energy of $0.7 \mathrm{MeV}$. Figure 4.6 shows a TOF spectrum obtained with this source when the scintillators are $20 \mathrm{~cm}$ apart. In this measurement, the PuLi source was enclosed in $1 \mathrm{~cm}$ of lead to reduce the gamma-ray flux from this source that produces excessive counting dead time in the data-collection system. For comparison, Figure 4.7 shows data obtained using an unshielded $\mathrm{PuBe}$ source (4.5 MeV average neutron energy) for approximately the same number of neutrons incident on the detector. The neutron detection efficiency is lower with the PuLi source because less energy is available for deposition in the scintillators. As mentioned previously, sources producing low-energy neutrons can be desirable for inducing fission while not interfering with the measurement of the more energetic fission neutrons.

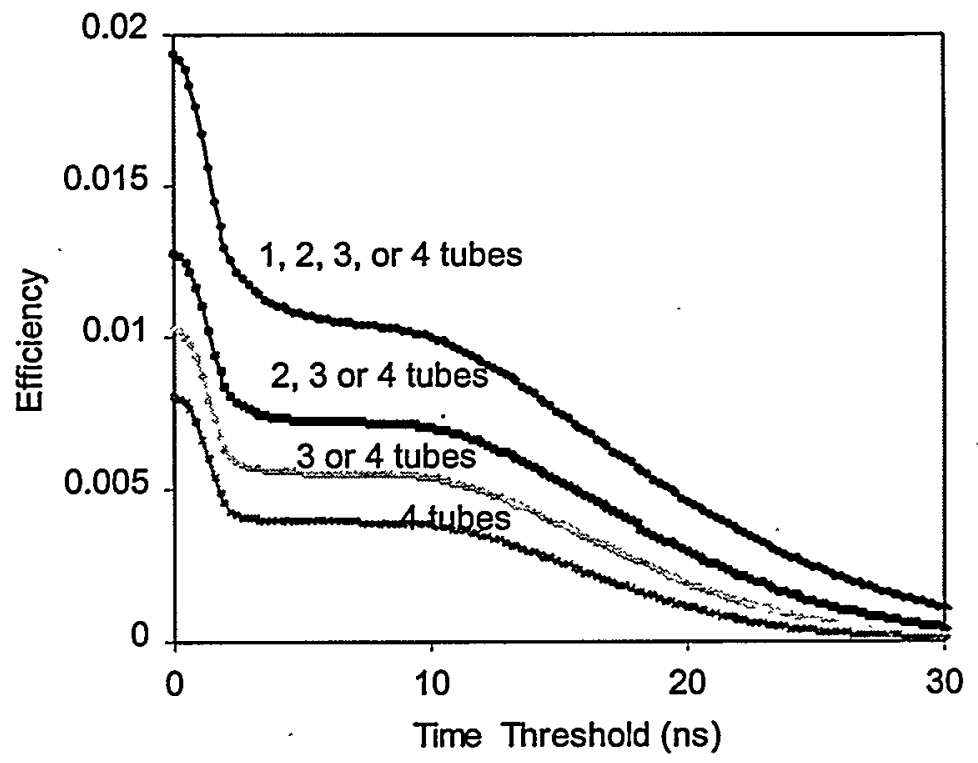

Figure 4.5. Neutron Detection Efficiency Dependence on Time. Threshold and Number of Tubes Detecting Events 


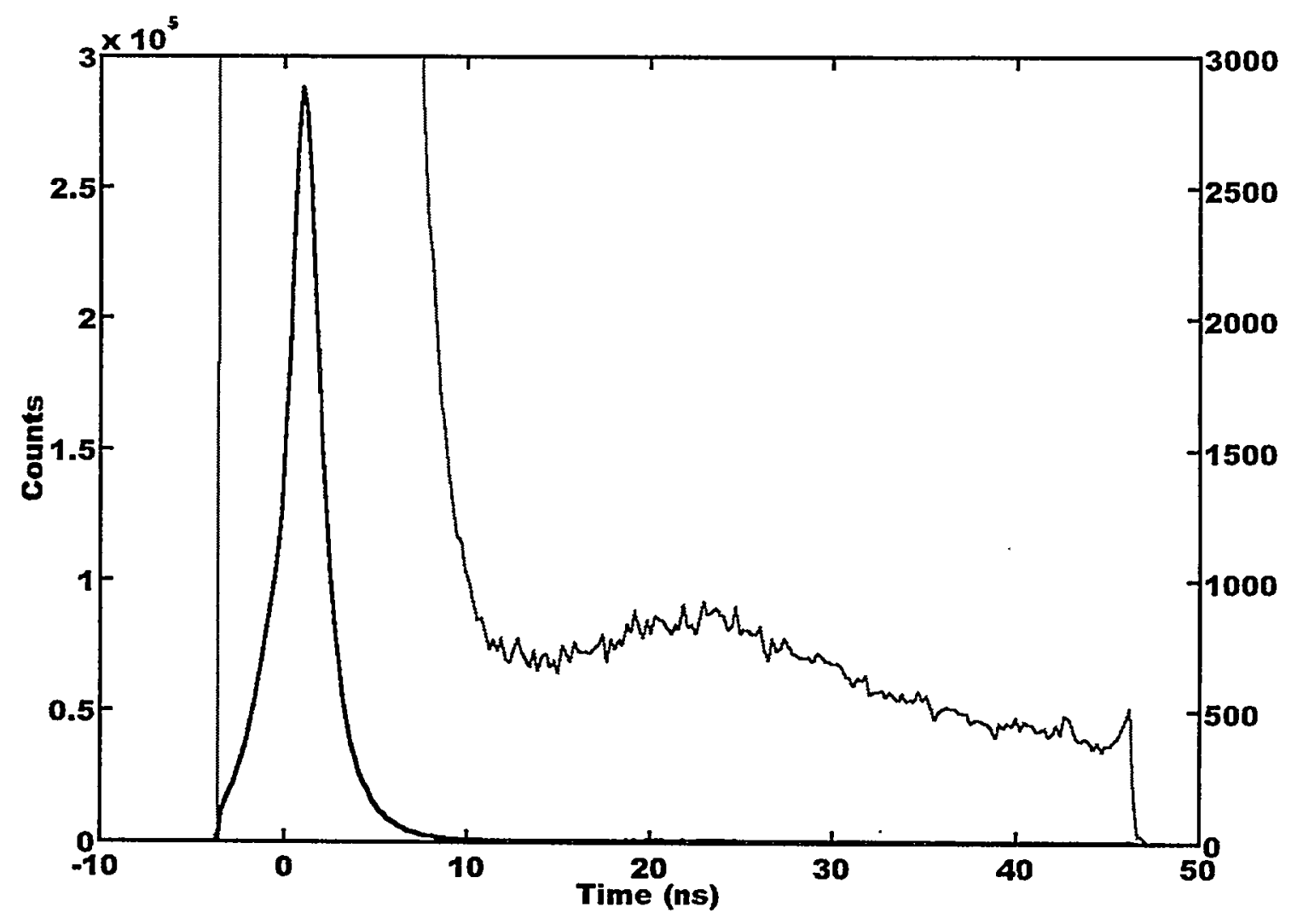

Figure 4.6. Time-of-Flight Spectrum Obtained Using PuLi Neutron Source, which Emits Neutrons with an Average Energy of $0.7 \mathrm{MeV}$. The light curve contains the same data plotted on a more sensitive scale (right side) showing the neutron peak. Scintillator separation distance $=20 \mathrm{~cm}$.

Comparing Figure 4.7 with Figure 4.3 shows the effect of the scintillator separation distance on the TOF spectra obtained using the PuBe source. For a gap of $30 \mathrm{~cm}$ (Figure 4.3), the neutron peak occurred around $18 \mathrm{~ns}$, whereas for the 20-cm gap (Figure 4.7), it occurred at about 11 ns.

TOF measurements offer the possibility of being able to distinguish neutron sources based on the energy of the neutrons. Although neutron sources are not monoenergetic, they can be characterized broadly according to their neutron energy. For example, $\mathrm{PuBe}$ sources emit neutrons with an average energy of about $4.5 \mathrm{MeV}$, while fission-neutron sources emit neutrons with an average energy of about $2 \mathrm{MeV}$. Table 4.1 lists several neutron sources and their energies. 


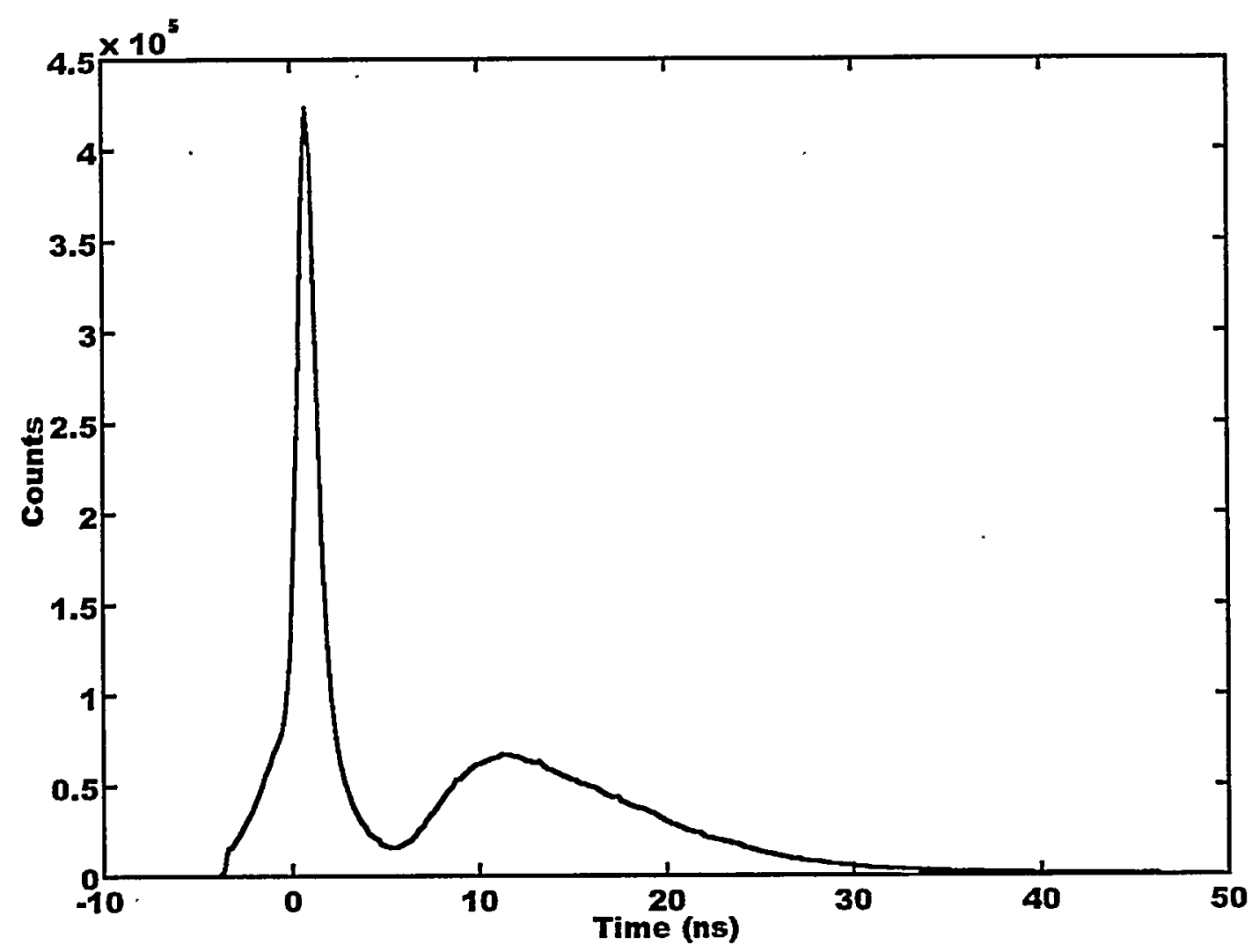

Figure 4.7. Time-of-Flight Spectrum for PuBe Source, which Emits Neutrons of Average Energy $4.5 \mathrm{MeV}$. Scintillator separation distance $=20 \mathrm{~cm}$.

Table 4.1. Neutron-Emitting Sources and Energies

\begin{tabular}{|l|c|c|c|}
\hline Source & Type & Avg. Alpha Energy (MeV) & Avg. Neutron Energy (MeV) \\
\hline${ }^{238} \mathrm{PuBe}$ & $(\alpha, \mathrm{n})$ & 5.49 & 4.5 \\
\hline${ }^{241} \mathrm{AmBe}$ & $(\alpha, \mathrm{n})$ & 5.48 & 5.0 \\
${ }^{252} \mathrm{Cf}$ & spontaneous fission & n.a. & 2.1 \\
${ }^{238} \mathrm{PuLi}$ & $(\alpha, \mathrm{n})$ & 5.49 & 0.7 \\
${ }^{238} \mathrm{PuO}_{2}$ & $(\alpha, \mathrm{n})$ & 5.49 & 2.0 \\
${ }^{230} \mathrm{PuF}_{4}$ & $(\alpha, \mathrm{n})$ & 5.15 & 1.4 \\
${ }^{241} \mathrm{AmLi}$ & $(\alpha, \mathrm{n})$ & 5.48 & 0.3 \\
\hline
\end{tabular}

A TOF spectrum should be useful in providing information on the energy spectrum from an unknown source, such as a neutron-emitting weapon component in a sealed container. For example, it may be possible to distinguish a fission spectrum from either a high-neutron-energy $(\alpha, n)$ source such as PuBe or AmBe and a low-energy $(\alpha, n)$ source such as PuLi or AmLi. 


\subsection{Detailed Analysis of TOF Spectra}

The following sections provide an alternative, more detailed analysis of TOF data obtained using the scintillator configuration shown in Figure 4.1 with the scintillator blocks separated by a distance of $20 \mathrm{~cm}$. Measurements were made on five neutron sources with differing energy spectra, ${ }^{137} \mathrm{Cs}$ gamma source, and backgrounds. All sources were measured using discriminator settings of $65 \mathrm{mV}$ and $100 \mathrm{mV}$ on the signals from the PMTs. In addition, the ${ }^{252} \mathrm{Cf}$ source was measured at a discriminator setting of $150 \mathrm{mV}$. Larger discriminator settings require more light detection by the PMTs and generally correspond to larger energy transfer from the neutron to the scintillator.

A detailed analysis of each time-of-flight spectrum was performed to extract the energy spectrum of the scattered neutrons. The first step in the analysis was to normalize the background TOF spectrum to the same counting time interval as the spectrum of interest and then to subtract the background. The sources were generally strong enough such that this correction had very little impact on the data.

The second step was to fit the sum of a narrow Gaussian peak and a wider Gaussian peak to the prompt coincidence peak due to gamma interactions. The fitted region was arbitrarily chosen to be $\pm 2.2 \mathrm{~ns}$ around the prompt peak. The fitted Gaussian peaks were subtracted from the background-subtracted data only over the region of the fit. In actuality, the fitted region had no influence on the time spectrum of scattered neutrons because only neutrons of greater than $40 \mathrm{MeV}$ were in this region.

The third step in the analysis was to subtract a constant level of accidental coincidences from every channel in the spectrum. The accidental coincidence rate was assumed to be that of the channel with the lowest counts in the vicinity of the longest time intervals (i.e., about $0.17 \mathrm{MeV}$ neutrons). For lack of more detailed knowledge, the accidental rate was assumed to be constant over the time range of interest $(50 \mathrm{~ns})$. This correction had a major influence on the TOF spectra for long time intervals.

The fourth step in the analysis was to correct the short time intervals for an assumed exponential decrease in the prompt coincidence peak due to gammas (the "false" neutron counts mentioned in Section 4.0). The exponential was normalized to the intensity of the spectrum at the boundary of the region fit by the Gaussians. The slope of the exponential was varied to give a smooth drop-off of the neutron TOF spectrum as it approached the prompt peak. The exponential shape of the tail of the prompt gamma peak is only an approximation. The resulting neutron TOF spectrum was very sensitive to this correction in the neutron energy region above about $3 \mathrm{MeV}$-especially for the sources that had a high ratio of gammas to neutrons.

An example of the corrections used in the analysis of the PuLi data taken with the discriminators at $65 \mathrm{mV}$ is shown in Figure 4.8. In this figure the prompt peak due to gammaray events is located at channel 212 , and the neutron-related events are to the left of this peak at the lower channel numbers because the reversal of start and stop signals during data acquisition. 
To convert the TOF spectrum to the energy spectrum of scattered neutrons, we use the relation given above (page 4.6) for neutron energy as a function of velocity. A significant problem is the uncertainty in the distance the neutron actually travels between collisions. For simplicity, we have assumed that the average distance traveled is the minimum distance from the midpoint of the first detector to the midpoint of the second detector $(25 \mathrm{~cm})$. Besides the uncertainties due to the thickness of the detectors $( \pm 2 \mathrm{~cm}$ in the thinner detector, $\pm 3 \mathrm{~cm}$ in the thicker detector), it is most likely that the neutrons travel a slanted path between the two detectors. Our assumed distance of $25 \pm 5 \mathrm{~cm}$ is therefore an underestimate of the actual distance. In the results that follow, we have made no corrections for the distribution of neutron path lengths, but this should be acceptable because our interest is in the relative spectral shapes from different sources; rather than the absolute TOF spectra.

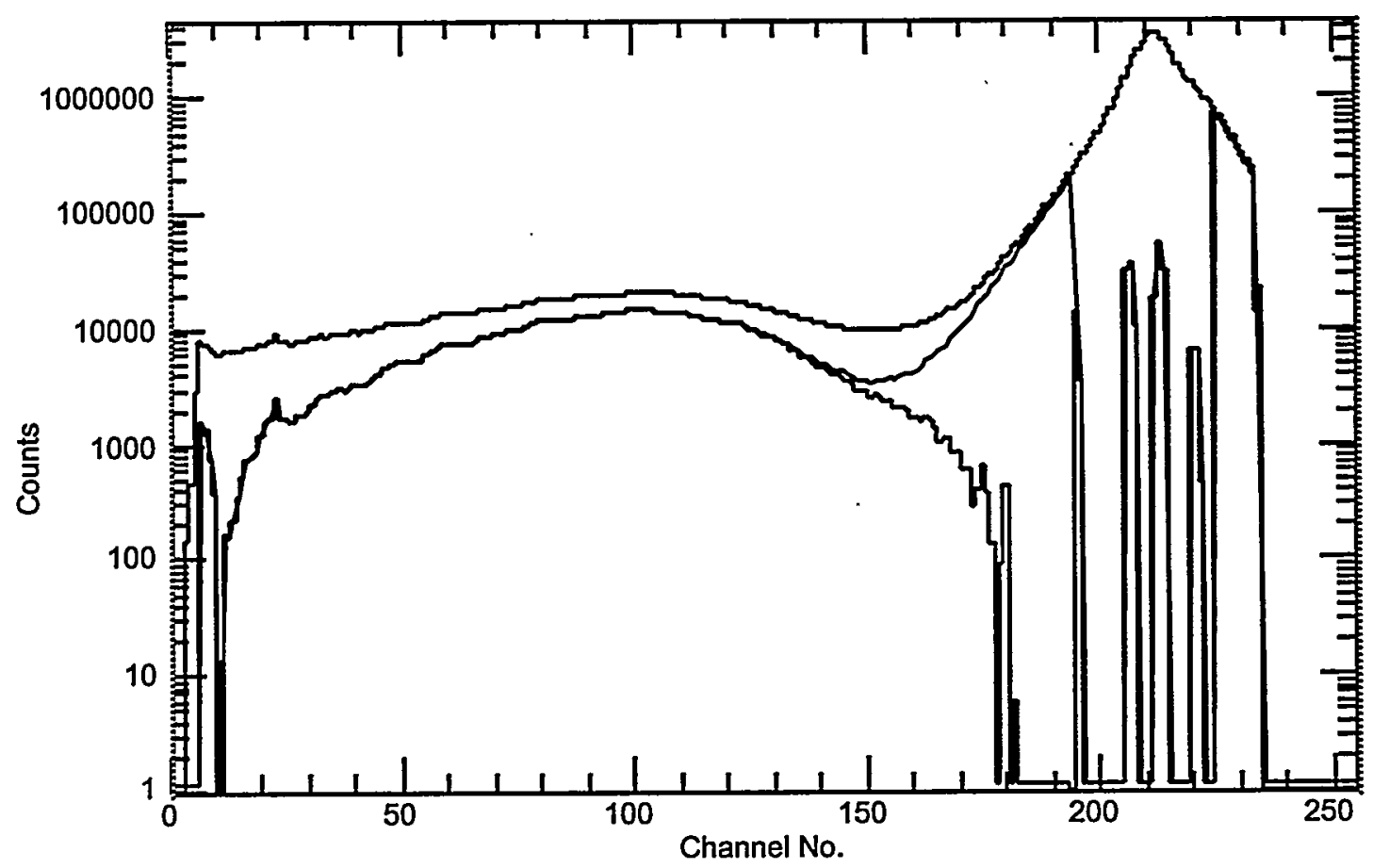

Figure 4.8. TOF Spectra for PuLi. The upper histogram is raw TOF spectrum for PuLi source with discriminators at $65 \mathrm{mV}$. The middle curve is the same data after subtracting the background, the prompt peak, and the accidentals. The lower histogram shows the result of subtracting an exponential tail due to the prompt peak. The region from Channel 10 to 180 of the lower histogram is the resulting neutron TOF spectrum. 


\subsection{TOF and Energy Spectra}

The effect of the various discriminator settings is illustrated in Figure 4.9 for the TOF spectra for the ${ }^{252} \mathrm{Cf}$ source. The spectra have been normalized to the channel with maximum counts. However, the trend is for the yield to decrease as the discriminators are raised, and there is a slight shift towards more energetic neutrons. The corresponding energy spectra are shown in Figure 4.10. The discriminators have a small effect on the shape of the energy spectra, but they have a significant effect on the detection efficiency, as will be discussed later.

In Figure 4.11, we compare the measured energy spectrum for scattered neutrons from
the ${ }^{252} \mathrm{Cf}$ source to the calculated neutron emission spectrum of the source approximated by a Maxwellian distribution with an average energy of $2.14 \mathrm{MeV}$. The peak of the scattered distribution is at roughly half the energy of the peak in the source spectrum. This is consistent with the expectation that the highest efficiency for the TOF detection system is when the scattered neutron deposits equal excitation energy in each scintillator.

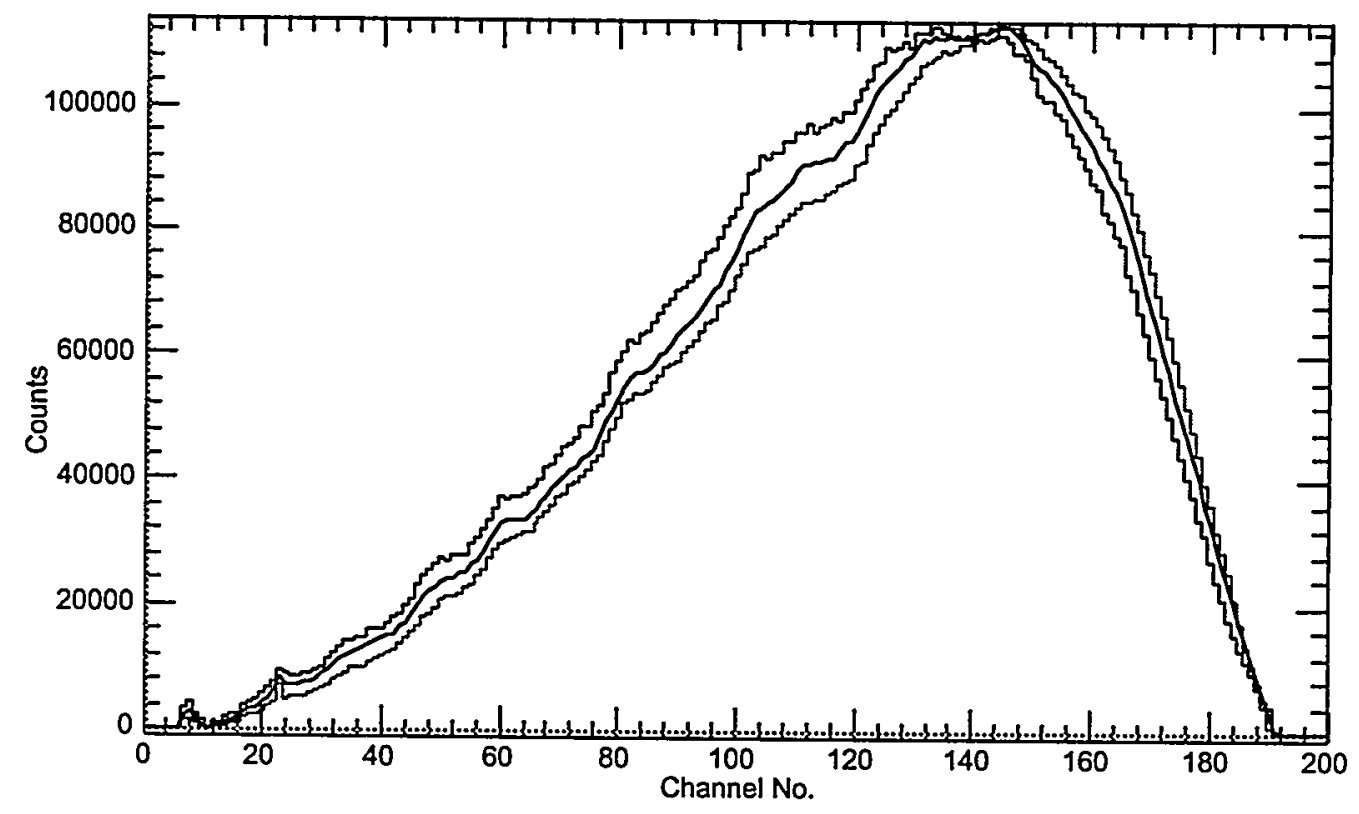

Figure 4.9. TOF Spectra (Normalized) for the ${ }^{252} \mathrm{Cf}$ Source at Three Different Discriminator Settings. The leftmost histogram is for the discriminator at $65 \mathrm{mV}$. The smooth curve is for the discriminator at $100 \mathrm{mV}$. The rightmost histogram is for the discriminator at $150 \mathrm{mV}$. 


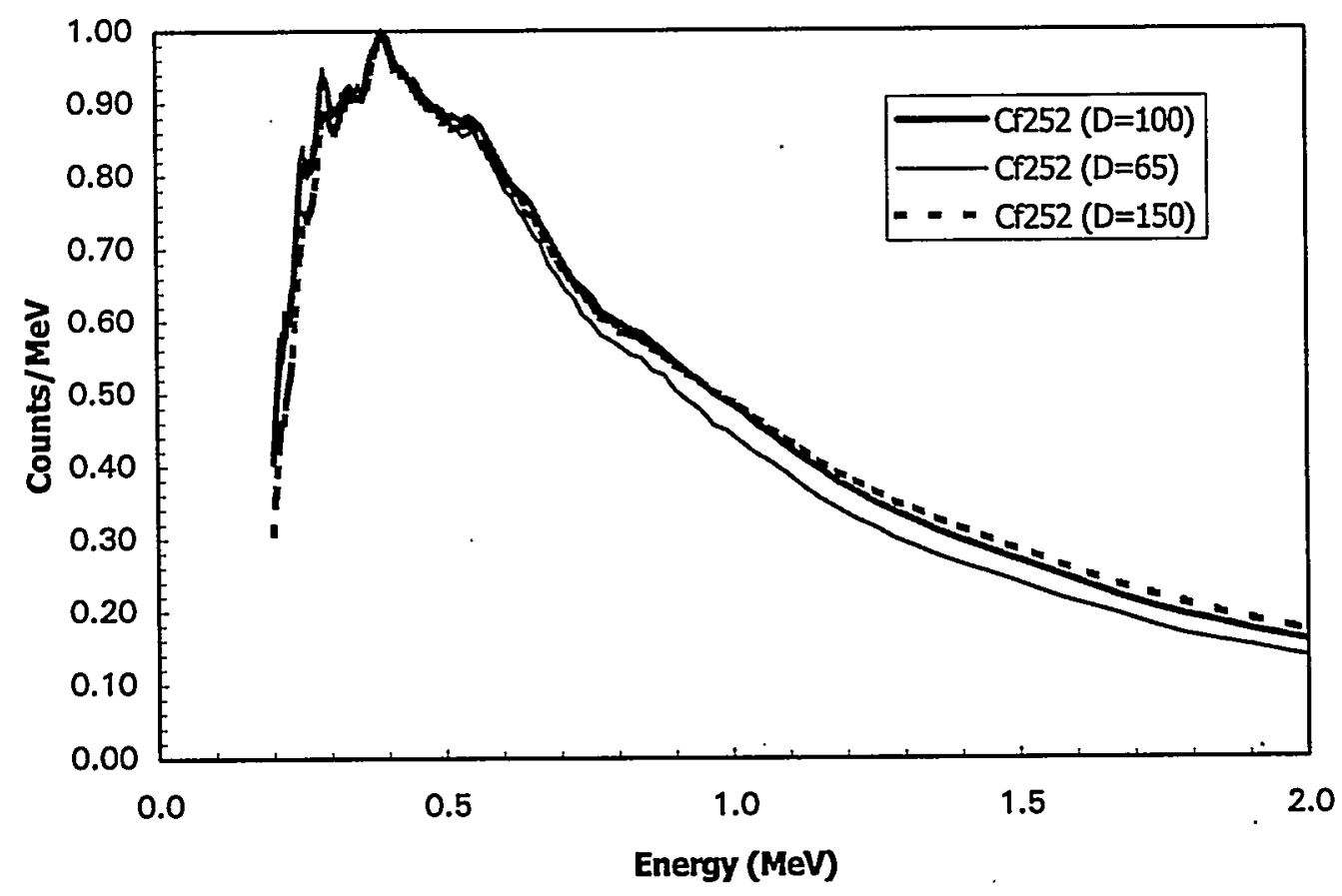

Figure 4.10. Energy Spectra for Scattered Neutrons from ${ }^{252} \mathrm{Cf}$ Source at Various Discriminator Settings. The spectra are decreasing smoothly beyond the $2 \mathrm{MeV}$ shown in the figure.

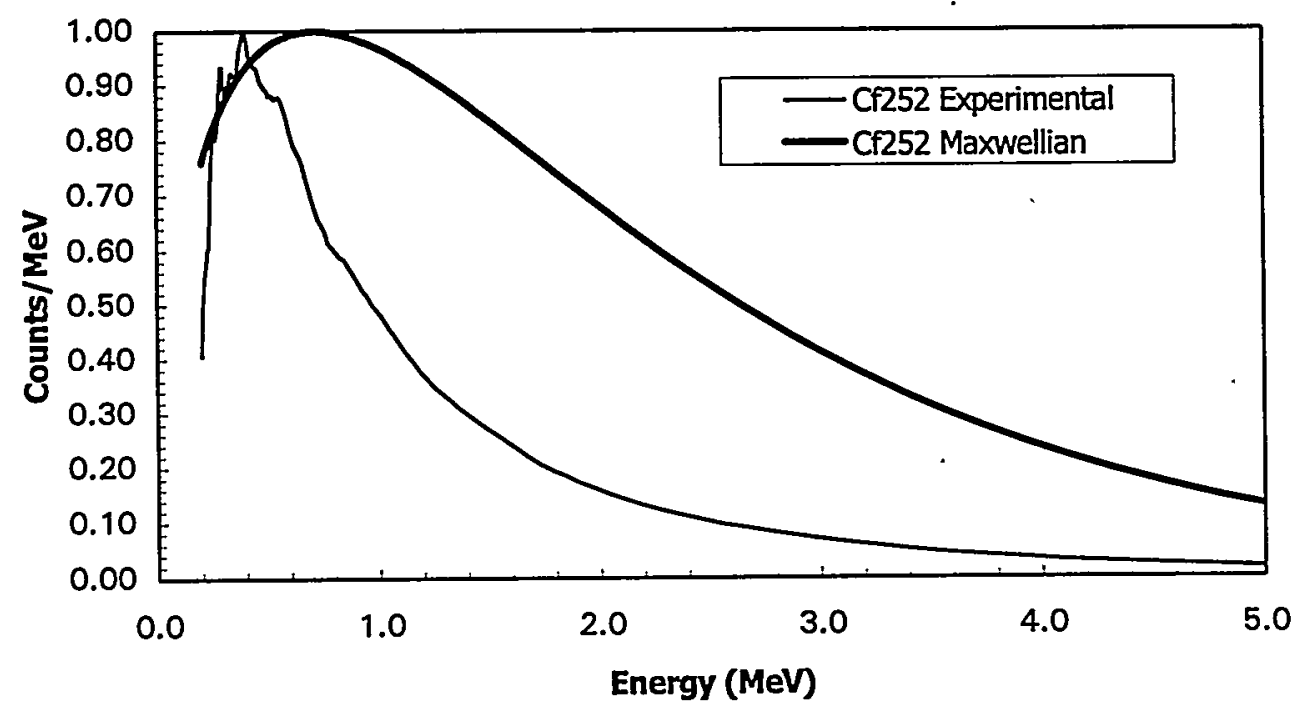

Figure 4.11. Energy Spectra for ${ }^{252} \mathrm{Cf}$. Heavy curve is calculated energy spectrum for neutron emission from the ${ }^{252} \mathrm{Cf}$ source based on a Maxwellian distribution with average energy of $2.14 \mathrm{MeV}$. Light curve is experimental energy spectrum for scattered neutrons in the TOF detector with a discriminator of $100 \mathrm{mV}$. 
One of the key questions in this work was whether the TOF technique would be able to distinguish different types of neutron sources based on their neutron-energy spectrum. Alpha sources containing Be give neutron spectra with high average energies of about $4.5 \mathrm{MeV}$. Alpha sources with Li give neutron spectra with low average energies of less than $1.0 \mathrm{MeV}$. Spontaneous fission sources give average neutron energies of about $2.0 \mathrm{MeV}$. In Figure 4.12, we compare the TOF spectra for three sources with different neutron emission spectra. Note that the TOF spectra are very different for each source, which confirms that the TOF technique can easily distinguish these different sources provided the energy spectra of the sources are not degraded by a moderator between the source and the detector.

The PuBe source emits neutrons due to $(\alpha, n)$ reactions on $\mathrm{Be}$. Only single neutrons are emitted per event, and the neutron-emission spectrum extends up to $10 \mathrm{MeV}$. The TOF spectrum for the scattered neutrons shown in Figure 4.12 has a distribution shifted toward short time intervals (high channel numbers) and correspondingly high energies.

The ${ }^{252} \mathrm{Cf}$ source is an example of a source that emits neutrons only by spontaneous fission. Each spontaneous fission event in ${ }^{252} \mathrm{Cf}$ has an average neutron multiplicity of 3.76 and an average gamma ray multiplicity of about 8 . Thus the TOF spectrum could in principle include data corresponding to "start" and "stop" events arising from different neutrons or gamma rays interacting in the two scintillators in addition to events due to scattering of a single neutron. However, there are three factors that reduce the probability of multiple particle events interfering with the ${ }^{252} \mathrm{Cf}$ TOF data. First, the source-to-detector distance of $30 \mathrm{~cm}$ gives only a 0.08 probability of a particle reaching the front detector due to the geometry. Second, the gamma rays have a small probability of giving pulses large enough to trigger the discriminators. Third, the TOF technique preferentially detects higher energy neutrons so that second and third neutrons from the source are likely to have lower energies and lower detection probabilities. The TOF spectrum for ${ }^{252} \mathrm{Cf}$ shown in Figure 4.12 has the broadest distribution and is shifted toward long time intervals.

The ${ }^{239} \mathrm{PuO}_{2}$ source is a mixture of neutrons from spontaneous fission and $(\alpha, n)$ reactions on oxygen. The isotopic composition of the source was used to determine the relative contribution from these two types of neutrons as shown in Table 4.2. Note that there are about equal contributions from each type. The energy spectrum of the spontaneous fission component of this source is very similar to the energy spectrum from ${ }^{252} \mathrm{Cf}$. However, the neutron energy spectrum for $(\alpha, n)$ reactions on oxygen has a distribution that only goes up to about $3.5 \mathrm{MeV}$ and has a peak at about $2.5 \mathrm{MeV}$. The TOF spectrum for the ${ }^{239} \mathrm{PuO}_{2}$ source shown in Figure 4.12 is intermediate between the $\mathrm{PuBe}$ and the ${ }^{252} \mathrm{Cf}$ spectra. It has a relatively narrow distribution like the $\mathrm{PuBe}$ source, but is shifted to lower energies. 


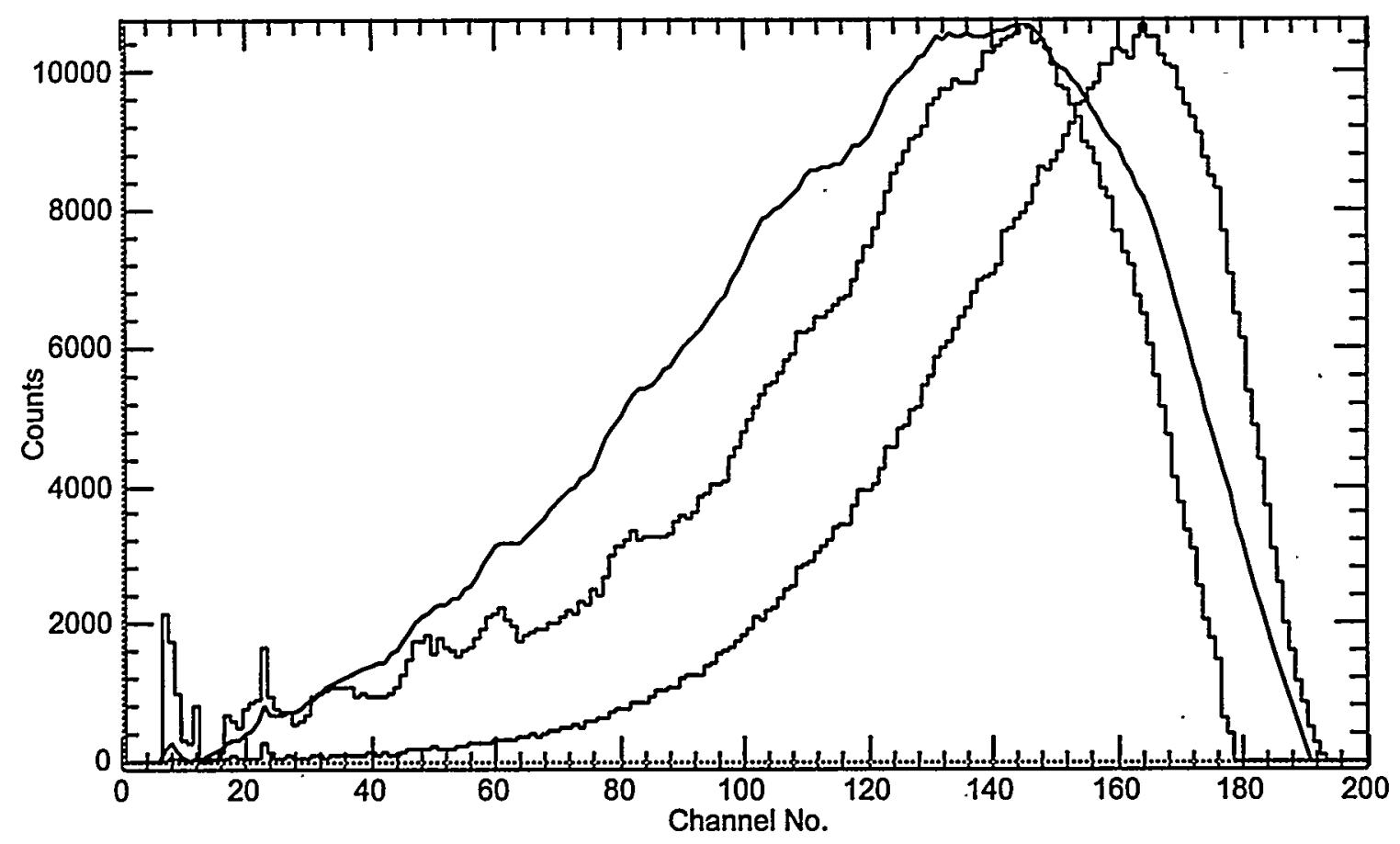

Figure 4.12. TOF Spectra for $\mathrm{PuBe},{ }^{252} \mathrm{Cf}$, and ${ }^{239} \mathrm{PuO}_{2}$ Sources with Discriminators at $100 \mathrm{mV}$. Rightmost histogram is PuBe. Smooth curve is ${ }^{252} \mathrm{Cf}$. Leftmost histogram is $\mathrm{PuO}_{2}$. Each spectrum has been normalized to its channel with the maximum count.

Table 4.2. Neutron Emission from ${ }^{239} \mathrm{PuO}_{2}$ Source

\begin{tabular}{cccrr} 
Pu Isotope & Wt. $(\mathbf{g})$ & Spont. Fission & $(\boldsymbol{\alpha}, \mathbf{n})$ & Total \\
\hline 238 & 0.0194 & 50 & 248 & 298 \\
239 & 93.908 & 2 & 4376 & 4378 \\
240 & 5.681 & 5795 & 966 & 6761 \\
241 & 0.198 & 0 & 0 & 0 \\
242 & 0.021 & 37 & 0 & 37 \\
\hline Total & & 5884 & 5590 & 11474
\end{tabular}

The energy spectra corresponding to the TOF spectra shown in Figure 4.12 are given in Figure 4.13 for the energy region from 0.2 to $2.0 \mathrm{MeV}$. The differences in the TOF spectra are equally well defined in the energy spectra for the three sources. A puzzling feature of the ${ }^{252} \mathrm{Cf}$ and $\mathrm{PuO}_{2}$ spectra is the rather narrow peaks between 0.2 and $0.4 \mathrm{MeV}$ that are not seen in the $\mathrm{PuBe}$ spectra. These peaks tend to be observed in the sources with weaker neutron emission rates. Some of the peaks occur at the same energy in different spectra, but not all peaks are in every spectrum. These peaks are not seen in the background spectra. The origin of these peaks is not understood, but they may be related to gamma interactions from the weaker sources. 


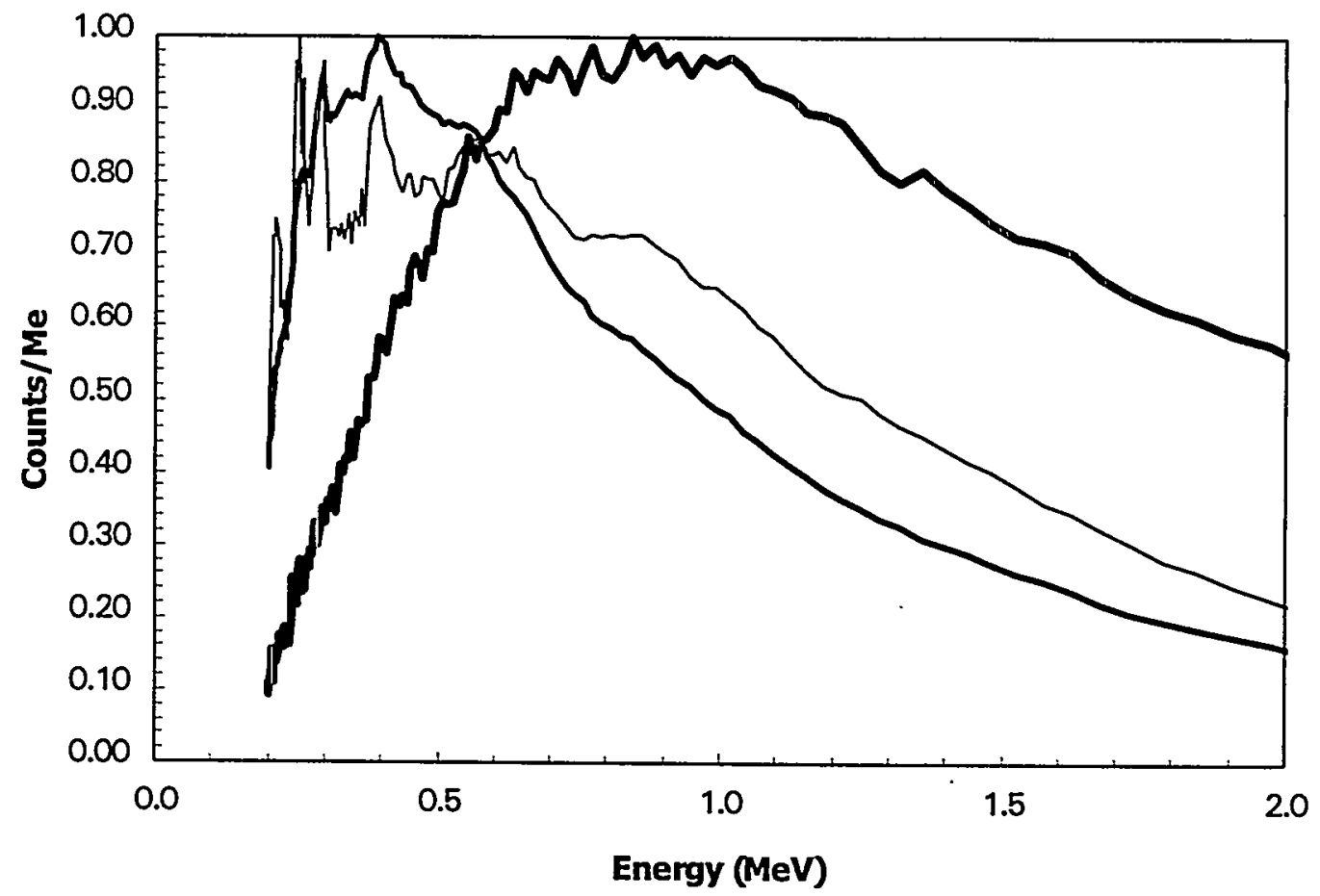

Figure 4.13. Energy Spectra of Scattered Neutrons with Discriminator at $100 \mathrm{mV}$. Heavy curve $=\mathrm{PuBe}$. Medium curve $={ }^{252} \mathrm{Cf}$. Light curve $=\mathrm{PuO}_{2}$.

The energy spectra of scattered neutrons from the AmLi and PuLi sources are shown in Figure 4.14. The narrow peaks below $0.4 \mathrm{MeV}$ show up strongly in the PuLi source, but are not so obvious in the AmLi source. The average energy for the PuLi source $(0.7 \mathrm{MeV})$ is higher than the average energy for the AmLi source (0.3) MeV as stated in Reilly et al. (1991). However, the spectrum of scattered neutrons from PuLi is clearly shifted to lower energies than the spectrum from AmLi. The peak in the spectrum for $\mathrm{AmLi}$ is at remarkably high energy and is comparable to the peak in the ${ }^{252} \mathrm{Cf}$ distribution. Previous measurements of the neutron spectrum from AmLi show two narrow peaks below $0.2 \mathrm{MeV}$ and a distribution that extends up to $1.5 \mathrm{MeV}$ (Weaver et al. 1982). The average energy as measured by Weaver et al. was 0.54 $\mathrm{MeV}$, which is higher than the average energy given by Reilly et al. It seems likely that because the TOF technique preferentially observes high-energy neutrons, the measured spectrum of scattered neutrons reflects the high-energy component of the AmLi emission spectrum.

\subsection{Fast-Neutron Detection Efficiency}

The detection efficiency for fast neutrons was calculated from the TOF spectrum remaining after the corrections for background, accidentals, and gamma peak. The integrated area of the TOF spectrum was divided by the neutron emission rate of the source, the counting time, and the fractional solid angle of the detector to the source. The resulting efficiency thus corresponds to the efficiency after the neutron has reached the front scintillator. Except for the $\mathrm{PuO}_{2}$ source, all the source emission rates were determined by calibration against a known 
standard source using an energy-independent detector in a scatter-free location. The strength of the $\mathrm{PuO}_{2}$ source was calculated on the basis of the isotopic composition as shown in Table 4.2. The fractional solid angle was the ratio of the area of the front face of the scintillator to the surface area of a sphere of $30-\mathrm{cm}$ radius. The results of all experiments at a source-to-detector distance of $30 \mathrm{~cm}$ are shown in Table 4.3 .

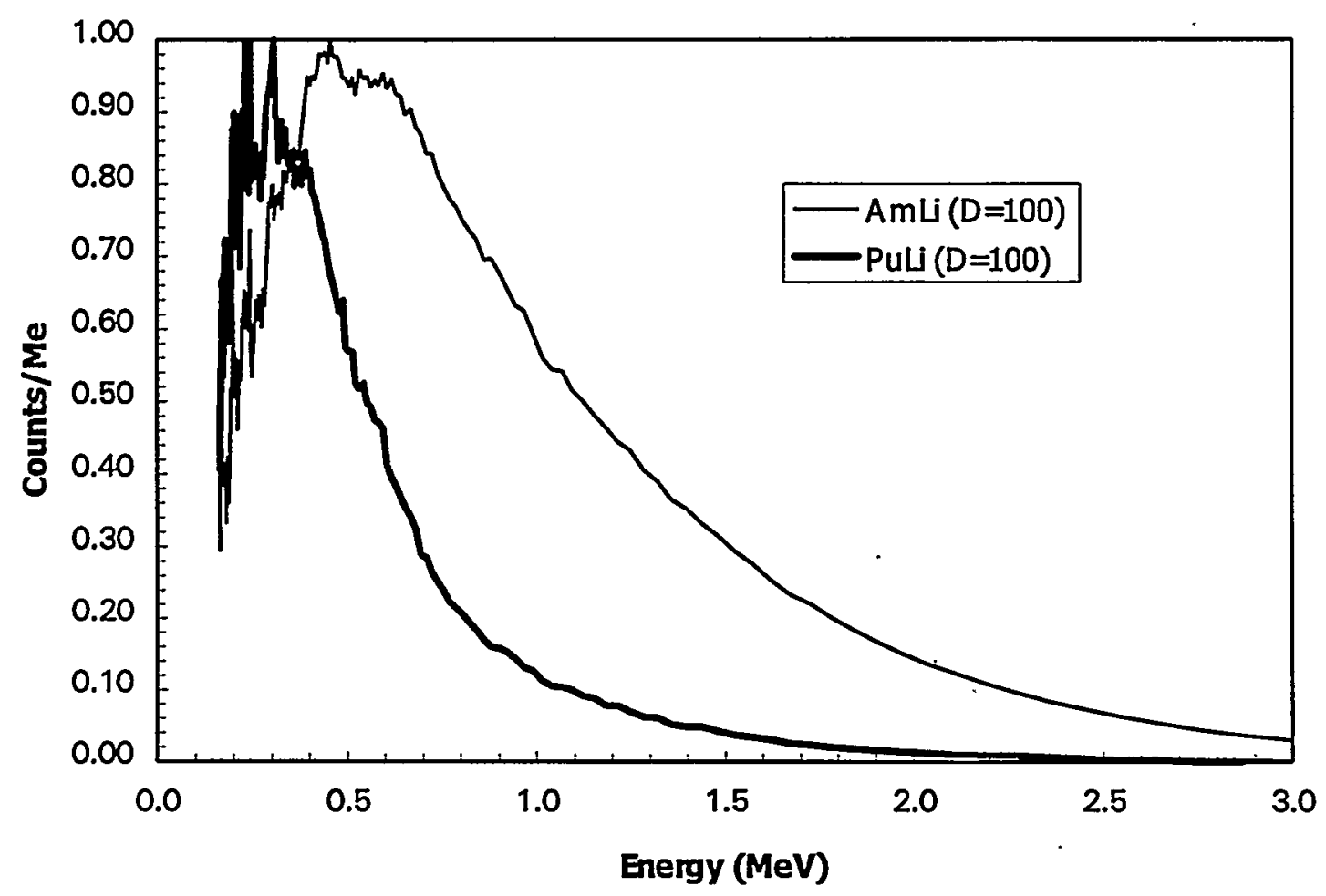

Figure 4.14. Energy Spectrum of Scattered Neutrons from AmLi and PuLi Sources with Discriminator at $100 \mathrm{mV}$. Light curve $=\mathrm{AmLi}$. Heavy curve $=\mathrm{PuLi}$.

Almost all runs were for counting times of $30,000 \mathrm{~s}$. Thus, the uncertainties based on counting statistics were negligible. However, the large corrections for background, accidentals, and gamma tail contributed to large systematic uncertainties in some cases. The uncertainties associated with these systematic corrections are difficult to estimate. For simplicity, we assumed that the uncertainty in the number of counts associated with a given correction was equal to $10 \%$ of the number of counts of the correction. The total uncertainty was the square root of the sum of the squared uncertainties for each of the individual uncertainties associated with the background correction, the accidental correction, and the exponential tail correction. The total uncertainty in the number of net counts was then used to calculate the uncertainty in the detection efficiency shown in Table 4.3. 
Table 4.3. Fast Neutron Detection Efficiency by Scattering TOF

\begin{tabular}{|c|c|c|c|c|c|c|c|}
\hline Source & Disc. & Run No. & Comment & $\begin{array}{c}\text { Source } \\
\text { Strength } \\
(\mathbf{n} / \mathbf{s})\end{array}$ & $\begin{array}{l}\text { Ave. n } \\
\text { Energy } \\
\text { (MeV) }\end{array}$ & $\begin{array}{c}\text { Efficiency } \\
(\%)\end{array}$ & $\begin{array}{l}\text { Sigma/ } \\
\text { Counts }\end{array}$ \\
\hline $\mathrm{AmLi}$ & 65 & $919-925$ & & 47,000 & 0.30 & $0.135 \pm 0.457$ & 3.388 \\
\hline PuLi & 65 & $810-811$ & & 658,000 & 0.70 & $0.036 \pm 0.008$ & 0.214 \\
\hline $\mathrm{PuO}_{2}$ & 65 & $965-967$ & $\mathrm{~Pb}$ & 11,500 & 2.00 & $1.121 \pm 0.988$ & 0.881 \\
\hline $\mathrm{Cf}$ & 65 & $940-942$ & & 336,000 & 2.14 & $4.372 \pm 0.210$ & 0.048 \\
\hline $\mathrm{Cf}$ & 65 & 970 & & 336,000 & 2.14 & $1.057 \pm 0.056$ & 0.053 \\
\hline $\mathrm{PuBe}$ & 65 & 804 & & 131,000 & 4.50 & $1.436 \pm 0.028$ & 0.019 \\
\hline AmLi & 100 & $926-933$ & & 47,000 & 0.30 & $0.196 \pm 0.070$ & 0.360 \\
\hline PuLi & 100 & $812-813$ & & 658,000 & 0.70 & $0.012 \pm 0.009$ & 0.743 \\
\hline $\mathrm{PuO}_{2}$ & 100 & $957-964$ & $\mathrm{~Pb}$ & 11,500 & 2.00 & $0.790 \pm 0.498$ & 0.631 \\
\hline $\mathrm{PuO}_{2}$ & 100 & $953-956$ & no $\mathrm{Pb}$ & 11,500 & 2.00 & $3.200 \pm 2.612$ & 0.816 \\
\hline $\mathrm{Cf}$ & 100 & $943-952$ & & 336,000 & 2.14 & $0.838 \pm 0.036$ & 0.043 \\
\hline $\mathrm{Cf}$ & 100 & $968-969$ & & 336,000 & 2.14 & $0.836 \pm 0.035$ & 0.042 \\
\hline PuBe & 100 & 805 & & 131,000 & 4.50 & $1.158 \pm 0.020$ & 0.017 \\
\hline $\mathrm{Cf}$ & 150 & $971-972$ & & 336,000 & 2.14 & $0.626 \pm 0.018$ & 0.029 \\
\hline${ }^{137} \mathrm{Cs}$ & 65 & $937-939$ & & 336,000 & & & 13.475 \\
\hline${ }^{137} \mathrm{Cs}$ & 100 & $935-936$ & & & & & 13.857 \\
\hline
\end{tabular}

The ratio of the uncertainty in the counts to the net counts is shown in the last column of Table 4.3. These ratios varied from a few percent for the strong, energetic sources $(\mathrm{PuBe}$ and ${ }^{252} \mathrm{Cf}$ ) to over $80 \%$ for weak or less energetic sources. The ratio of uncertainty to net counts is also shown for the ${ }^{137} \mathrm{Cs}$ gamma source. In this case, the corrections reduced the net neutron signals at all time intervals to a level that was insignificant compared to the uncertainty in the corrections.

For the ${ }^{252} \mathrm{Cf}$ source, we have efficiencies measured at three different discriminator settings. These efficiencies are shown in Figure 4.15. As expected, the efficiencies decrease as the discriminator is increased.

Although the scattering TOF technique is a powerful method to distinguish neutron sources with differing energy spectra, the efficiencies for fast-neutron detection are not as high as we had anticipated for the high-energy sources such as $\mathrm{PuBe}$ and ${ }^{252} \mathrm{Cf}$. The maximum efficiency observed in this work was about $1.4 \%$ for the PuBe source with the discriminator at $65 \mathrm{mV}$. Although higher efficiencies could be obtained by operating at lower discriminator settings, the contribution of gamma interactions increases rapidly as the discriminator is lowered, which makes the distinction between neutrons and gammas more difficult. With the discriminator set at $100 \mathrm{mV}$, the efficiency for detecting neutrons from the low-energy PuLi source was a factor of 100 lower than the efficiency for the PuBe source. This confirms our expectation that the scattering TOF technique can be used to monitor fission-spectrum neutrons in the presence of low-energy neutrons from an active interrogation source such as PuLi. 


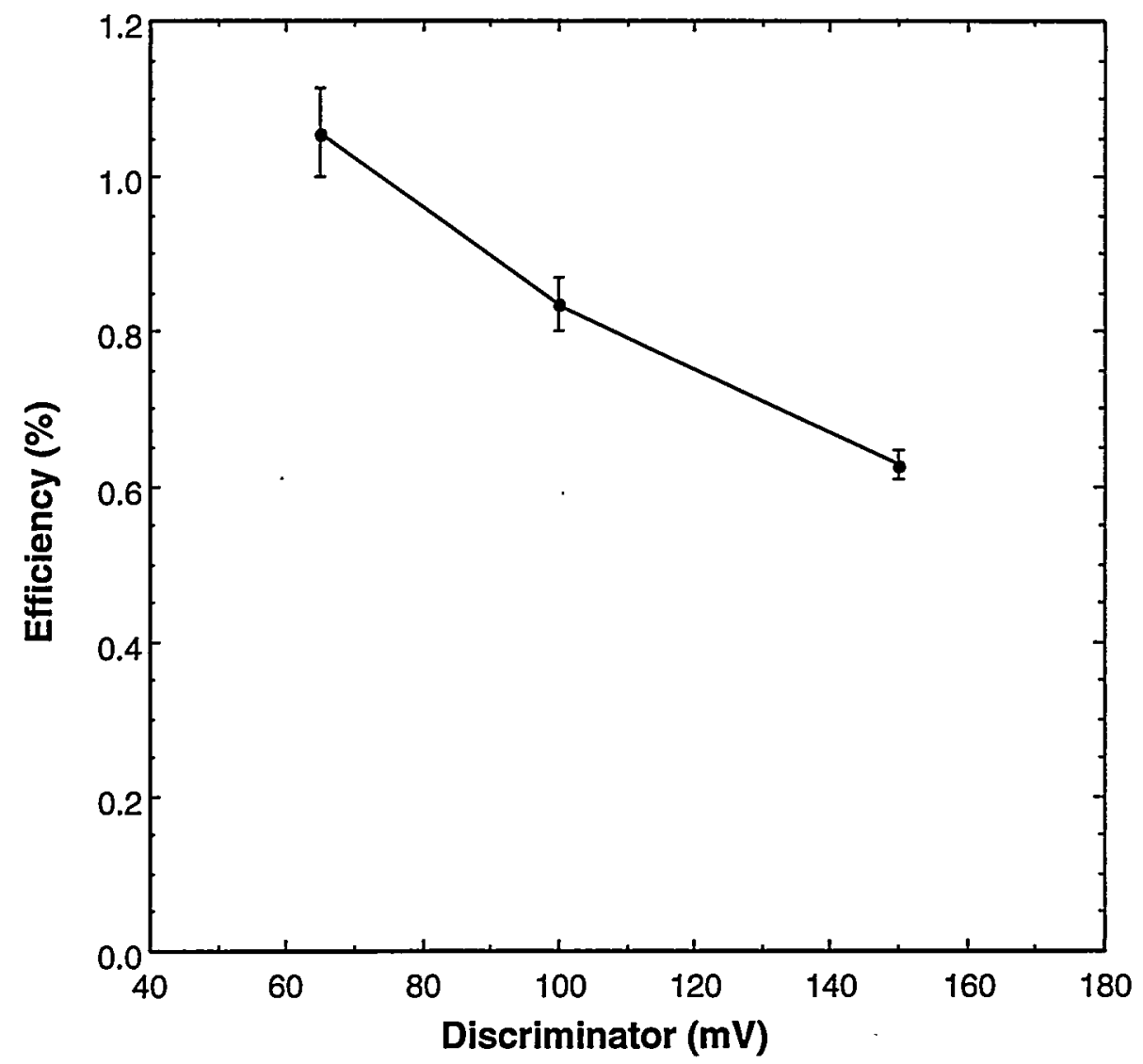

Figure 4.15. Fast Neutron Detection Efficiency by Scattering TOF for ${ }^{252} \mathrm{Cf}$ Source with Various Discriminator Levels. The lines are drawn merely to guide the eye.

\subsection{Analysis of TOF Versus PMT Multiplicity}

Besides obtaining TOF spectra as a function of discriminator setting, we also measured the TOF spectra as a function of the number of photomultiplier tubes (PMTs) in a given scintillator that gave a pulse above the discriminator threshold. These data were obtained in a two-dimensional array of TOF spectra versus the multiplicity signal from the constant fraction discriminator module (CF8000). The 2D arrays were recorded separately for the thin scintillator and the thick scintillator, but only the data from the thin scintillator are described here. The 2D arrays were analyzed offline by setting a window on the channels corresponding to only 1 PMT contributing to the event, only 2 PMTs contributing, only 3 PMTs, or only 4 PMTs. Our expectation was that scattering events that deposited a large amount of excitation would trigger several PMTs whereas if only a small amount of excitation energy was deposited, then perhaps only 1 PMT would be triggered. The PMT multiplicity then becomes another way of determining whether the event was caused by a high-energy neutron or a low-energy neutron. 
In Figure 4.16, we show the TOF spectra for the PuBe source with discriminator $=100 \mathrm{mV}$ for the case where only 1 PMT was triggered and the case where all 4 PMTs were triggered. For this high-energy source, the number of events involving all 4 PMTs was much greater than the number of events involving only 1 PMT. Similar data are shown in Figure 4.17 for the PuLi source. With this low-energy source, the number of events involving $1 \mathrm{PMT}$ is greater than the number of events involving 4 PMTs. Thus, the ratio of the number of events triggering 1 PMT to those triggering 4 PMTs is a very strong function of the energy spectrum of the source. For all the sources, the number of 2 PMT events and 3 PMT events was less than either the 1 PMT or 4 PMT events.

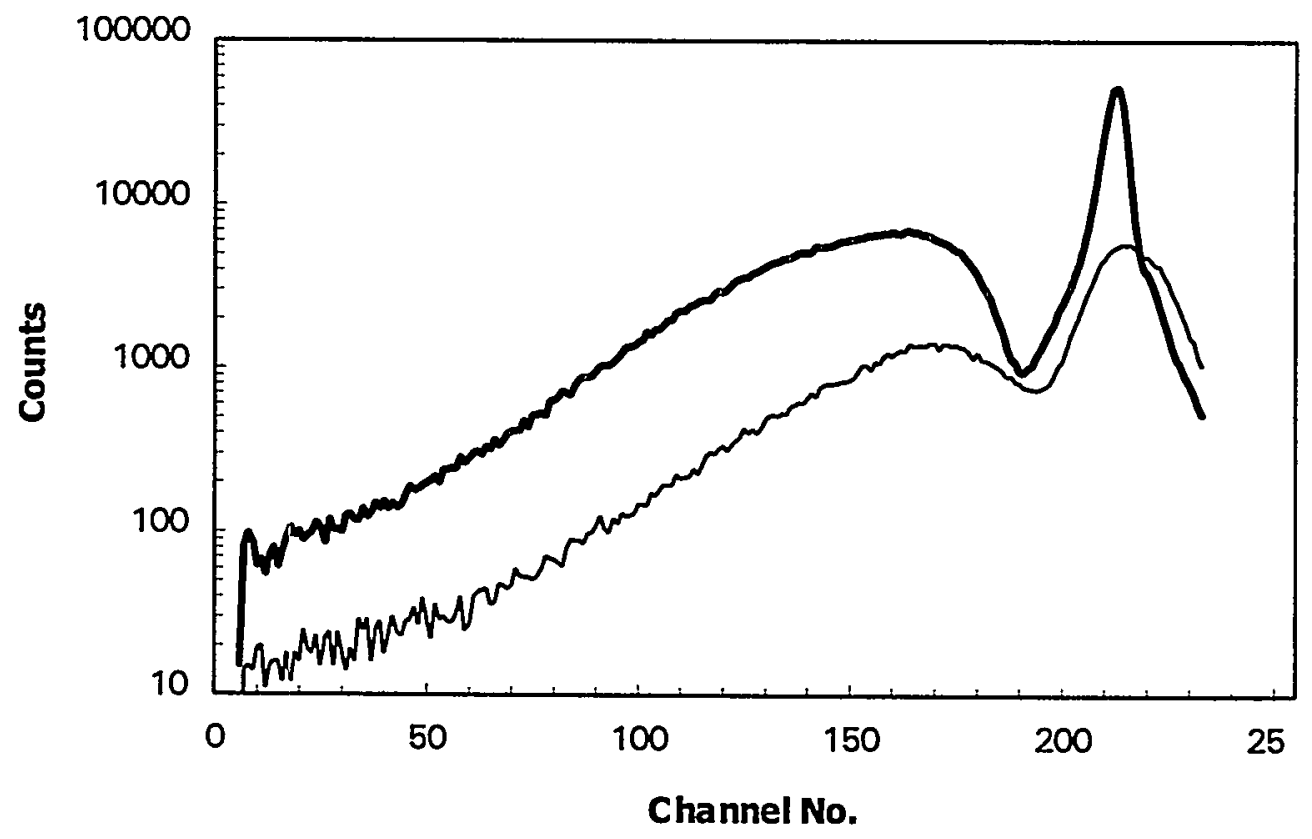

Figure 4.16. TOF Spectra for PuBe Source with Discriminator $=100 \mathrm{mV}$. Heavy curve $=\mathrm{TOF}$ spectrum when all 4 PMTs were triggered. Light curve $=$ TOF spectrum when only 1 PMT was triggered.

Table 4.4 gives the 1 PMT to 4 PMT ratio for all the sources measured here. Note that a complete stripping of the TOF spectrum was not performed for the data in Table 4.4. Only the accidental correction has been applied, so the ratios given in Table 4.4 are not quantitatively precise. However, they do illustrate the large differences in this ratio as a function of neutron energy. Note that the AmLi source has an anomalous ratio just as its scattered energy spectrum and detection efficiency were anomalous relative to the other sources. Unfortunately, searching for the cause of these anomalies is outside the scope of this work. 


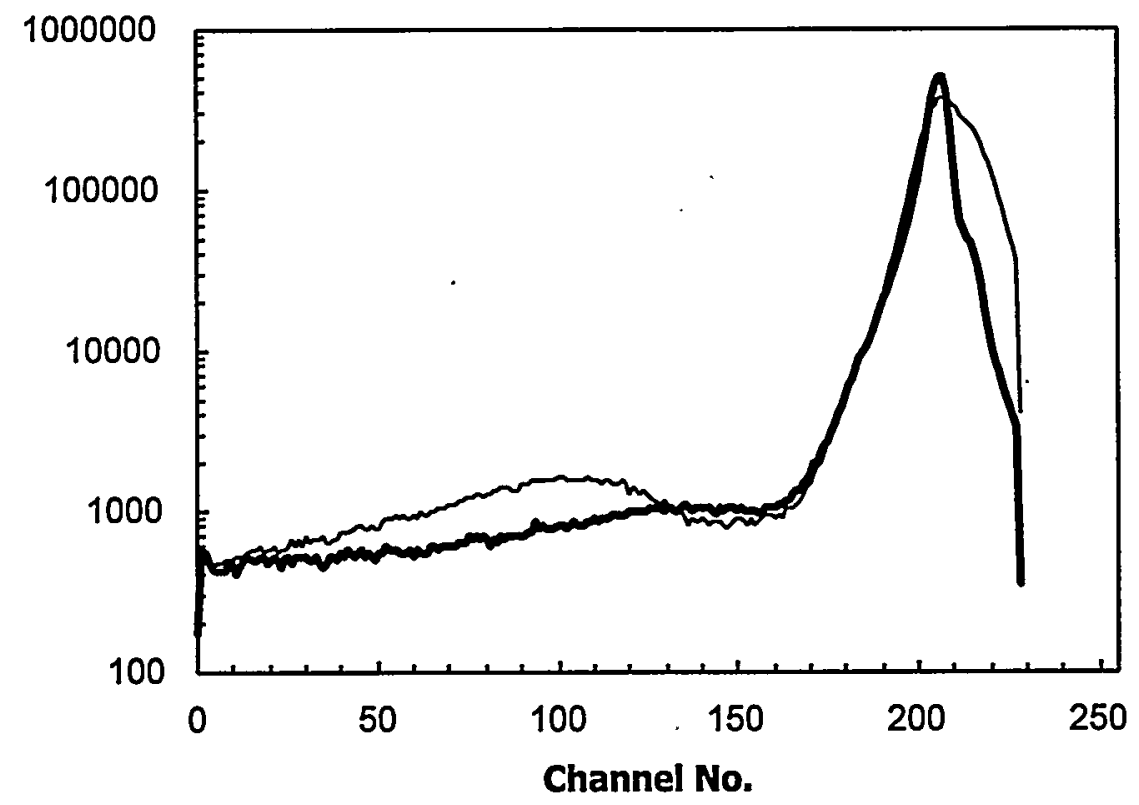

Figure 4.17. TOF Spectra for PuLi Source with Discriminator $=100 \mathrm{mV}$. Heavy curve $=\mathrm{TOF}$ spectrum when all 4 PMTs were triggered. Light curve $=$ TOF spectrum when only 1 PMT was triggered.

Table 4.4. Ratio of 1 PMT Events to 4 PMT Events for Various Sources with Discriminator at $100 \mathrm{mV}$

\begin{tabular}{ccc} 
Source & Ave. Energy (MeV) & Ratio 1 PMT/4 PMT \\
\hline $\mathrm{AmLi}$ & 0.3 & 0.25 \\
$\mathrm{PuLi}$ & 0.7 & 1.75 \\
$\mathrm{PuO}_{2}$ & 2.0 & 0.72 \\
${ }^{252} \mathrm{Cf}$ & 2.1 & 0.43 \\
$\mathrm{PuBe}$ & 4.5 & 0.18 \\
\hline
\end{tabular}




\subsection{Possible Future Work}

The method to detect fast neutrons based upon multiple scattering in a single piece of fast scintillator needs the development of a faster scintillator than is now commercially available. Adherent Technologies in Albuquerque, New Mexico, is working on new scintillators and may create one with sufficient performance to enable the pulse width to give a basis for distinguishing neutrons from gamma rays. If a new, fast scintillator becomes available, it should be tested for fast-neutron response using the pulse-width discrimination method.

TOF provides a presently available method for detecting fäst neutrons and discriminating them from gamma rays. The measured detection efficiency in the tested configuration was about $1 \%$. This efficiency is low for applications such as coincidence counting where coincident neutron flux is also low. Other applications, such as determining (at least coarsely) the energy of neutrons emitted from a sealed container, appear to be viable for the TOF technique.

Experiments described in this report show that the TOF method can distinguish between neutron spectra categories (such as fission spectra and $(\alpha, n)$ sources with a Be or Li target). Additional work could include a closer look at the TOF spectral differences between $\mathrm{Pu}$ metal and $\mathrm{PuO}_{2}$, including the use of additional sources with different ${ }^{240} \mathrm{Pu}$ contents. Future work could determine how much useful information on neutron energy is retained in the presence of some moderating material.

The equipment used in the present fast-neutron studies uses a large, light-tight box to enclose the plastic scintillators. If non-laboratory measurements are to be made in the future using TOF, the equipment will need to be made light-tight and more readily transportable. 


\subsection{Conclusion}

Fast neutrons that undergo multiple scattering in plastic scintillators offer the potential for discriminating between neutron and gamma-ray events based on a broader pulse width from the neutrons. Experimental results using gamma rays from ${ }^{137} \mathrm{Cs}$ and neutrons from ${ }^{252} \mathrm{Cf}$ were consistent with having broader pulses from neutrons, but the magnitude of the difference was too small to distinguish neutron and gamma-ray events on a pulse-by-pulse basis. The gamma-ray pulses were broader than expected, and no commercial plastic scintillator appears to have the necessary fast response to produce the needed short pulses from gamma-ray events.

An alternative approach for detecting fast neutrons used scintillators separated by an air gap to extend the travel time of neutrons between successive collisions. Scintillators in this configuration do not need a particularly fast response, and discrimination between neutrons and gamma rays is based on TOF. Experiments using two blocks of BC408 scintillator $(4 \times 30 \times 30 \mathrm{~cm}$ and $6 \times 30 \times 30 \mathrm{~cm}$ ) separated by gaps of 20 to $30 \mathrm{~cm}$ measured the efficiency for fast neutron detection from a ${ }^{239} \mathrm{PuBe}$ source to be about $1 \%$. The TOF measurement was also tested with sources that emitted neutrons of different energies. The TOF information, coupled with the multiplicity of photomultiplier tubes that detected the neutron events, provided a method for distinguishing high-energy neutrons from low-energy (not thermal) neutrons.

There are three main conclusions from the work with the neutron-scattering TOF technique.

1. We have demonstrated that the neutron-scattering TOF technique has about 100 times more efficiency for detecting fast neutrons from high-energy sources such as $(\alpha, n)$ reactions on $\mathrm{Be}$ than for fast neutrons from low-energy sources such as $(\alpha, n)$ reactions on $\mathrm{Li}$. The efficiency for neutrons from spontaneous fission is comparable to the efficiency for high-energy sources. This technique may thus find application where fissionable material is actively interrogated with low-energy neutrons. The induced fission neutrons can be counted even in the presence of the active source. The discrimination against gamma events can be greater than $10^{6}$.

2. The TOF technique requires time intervals of about $50 \mathrm{~ns}$ for the system tested here. To use this technique in a coincidence system with multiple modules would require a coincidence resolving time of about $50 \mathrm{~ns}$. This is far faster than the standard moderate and capture type of neutron detectors, but is longer than the few ns resolving time that could be obtained from a single plastic scintillator detector. A more serious problem for this technique when considering its use in neutron multiplicity detectors is the low efficiency $(1.4 \%)$ even for energetic neutrons from $(\alpha, n)$ reactions on $\mathrm{Be}$.

3. The neutron-scattering TOF technique is very successful in distinguishing neutron sources with different energy spectra. A possible application for this technique would be to distinguish $\mathrm{Pu}$ metal, which has only neutrons from spontaneous fission and $\mathrm{PuO}_{2}$, which has neutrons both from spontaneous fission and $(\alpha, n)$ reactions on oxygen. 


\subsection{References}

Briesmeister J. 1993. MCNP-A General Monte Carlo N-Particle Transport Code, Version 4a," LA-12625-M, Los Alamos National Laboratory, Los Alamos, New Mexico.

Hansen RR, PL Reeder, AJ Peurrung, and DC Stromswold. 1998. "Neutron-gamma discrimination in plastic scintillators," presented at IEEE Nuclear Science Symposium, Toronto, Nov. 1998, to appear in conference record.

Knoll GF. 1989. Radiation Detection and Measurement, ch. 15, Wiley, New York.

Peurrung AJ, CW Hubbard, RR Hansen, PE Keller, RA Craig, PL Reeder, WK Hensley, and DS Sunberg. 1997. Direct Fast-Neutron Detection: a Progress Report, PNNL-11783, Pacific Northwest National Laboratory, Richland, Washington.

Peurrung AJ, RR Hansen, PL Reeder, and DC Stromswold. 1998. Direct Fast-Neutron . Detection: a Progress Report, PNNL-11994, Pacific Northwest National Laboratory, Richland, Washington.

Reeder, PL, AJ Peurrung, RR Hansen, DC Stromswold, WK Hensley, and CW Hubbard. 1998. "Detection of fast neutrons in plastic scintillator using digital pulse processing to reject gammas," Nucl. Instr. and Meth. In Phys. Res. A 422, pp. 84-88.

Reilly D, N Ensslin, and H Smith, Jr., eds. 1991. Passive Nondestructive Assay of Nuclear Materials, ch. 16-17, LA-UR-90-732 and NUREG/CR-5550, Los Alamos National Laboratory, Los Alamos, New Mexico.

Vanier, PE, L Forman, and EC Selcow. 1995. "A thermal neutron imager using coded aperatures," Nuclear Materials Management, Proc. $35^{\text {th }}$ Annual Meeting, INMM, Palm Desert, 24, p. 842 .

Weaver, DR, JG Owen, and J Walker. 1982. "The Neutron Spectrum from a $5 \mathrm{Ci} \mathrm{Am/Li}$ Neutron Source," Nucl. Instrum. Meth., 198, 599. 


\section{Distribution}

No. of

Copies

\section{OFFSITE}

2 DOE Office of Scientific and Technical Communications

Michael O'Connell, NN-20

U.S. Department of Energy 1000 Independence Ave. SW

Washington, DC 20585-0420

David Spears (2), NN-20

U.S. Department of Energy 1000 Independence Ave. SW

Washington, DC 20585-0420

Paul Goldhagen

Environmental Measurements

Laboratory

201 Varik Street, $5^{\text {th }}$ Floor

New York, NY 10014-4811

John Mihalczo

Oak Ridge National Laboratory

P.O. Box 2009

Oak Ridge, TN 37831-8121

Bill Winn

Savannah River Technology Center

P.O. Box 616

Aiken, SC 29808
No. of

Copies

\section{ONSITE}

55 Pacific Northwest National

Laboratory

M. Bliss

P8-20

R. A. Craig

K5-25

G. B. Dudder (10)

$\mathrm{K} 6-48$

B. D. Geelhood

P8-08

A.J. Peurrung (15)

P8-08

P. L. Reeder (5)

P8-08

D. E. Robertson

P8-20

D. C. Stromswold (15)

P8-08

R. A. Warner

K6-48

Technical Report Files (5)

Distr. 1 